Projets

de paysage

\section{Projets de paysage}

Revue scientifique sur la conception et l'aménagement de l'espace

$16 \mid 2017$

Arbres et paysages

\title{
Évolution des paysages arborés dans les montagnes de Thừa Thiên Huế (Việt Nam)
}

Quelles incidences en matière de biodiversité et de services écosystémiques rendus aux ethnies minoritaires?

The Evolution of Tree Landscapes in the Mountains of Thừa Thiên Huế(Việt Nam) - The Impacts on Biodiversity and Ecosystemic Services Provided to Ethnic Minorities

\section{Amélie Robert}

\section{CpenEdition}

\section{Journals}

\section{Édition électronique}

URL : http://journals.openedition.org/paysage/6046

DOI : 10.4000/paysage.6046

ISSN : 1969-6124

\section{Éditeur :}

École nationale supérieure du paysage de Versailles-Marseille, Institut national des sciences appliquées Centre Val de Loire - École de la nature et du paysage, École nationale supérieure d'architecture et de paysage de Bordeaux, École nationale supérieure d'architecture et de paysage de Lille, Agrocampus Angers

\section{Référence électronique}

Amélie Robert, «Évolution des paysages arborés dans les montagnes de Thừa Thiên Huế (Việt Nam) », Projets de paysage [En ligne], 16 | 2017, mis en ligne le 05 juillet 2017, consulté le 30 avril 2020. URL : http://journals.openedition.org/paysage/6046; DOI : https://doi.org/10.4000/paysage.6046 


\section{Évolution des paysages arborés dans les montagnes de Thừa Thiên Huế (Việt Nam)}

Quelles incidences en matière de biodiversité et de services écosystémiques rendus aux ethnies minoritaires?

The Evolution of Tree Landscapes in the Mountains of Thừa Thiên Huế (Việt Nam) - The Impacts on Biodiversity and Ecosystemic Services Provided to Ethnic Minorities

\section{Amélie Robert}

1 Colonisation, guerre, collectivisation et, depuis 1986, période dite du « Renouveau » se sont succédé au cours de l'histoire vietnamienne contemporaine, du XIX siècle à nos jours À chacune de ces périodes, les pratiques sociales ont transformé les paysages, notamment ceux des montagnes. Peuplées par les ethnies minoritaires ${ }^{1}$, ces montagnes concentrent l'essentiel des superficies forestières du pays. Ces forêts ont été particulièrement affectées, sous le poids de la guerre, de l'exploitation forestière et de la progression d'une agriculture intensive. Les paysages ont ainsi évolué et l'environnement des sociétés montagnardes s'est transformé. Quelle place occupe l'arbre dans les paysages actuels et passés ? Quelles en sont les évolutions en termes de biodiversité - diversité des espèces végétales, considérée "comme le résultat d'une coévolution naturelle et sociale » (Simon, 2006) ? N’y a-t-il pas aussi des conséquences pour les montagnards? Si oui, quelles sont les incidences de ces dynamiques sur les usages, sur les « services » rendus à ces populations? Sont ici considérés les services écosystémiques, tels que définis par le Millenium Ecosystem Assessment (MEA, 2005) "benefits people obtain from ecosystems"-, surtout ceux dont les retombées sont directement perceptibles par les villageois, en l'occurrence les services d'approvisionnement et culturels ${ }^{2}$ Les premiers renvoient aux ressources qu'offrent les écosystèmes ; il s'agit par exemple, pour les écosystèmes forestiers, du bois fourni par les arbres et utilisé comme matériau de construction ou bien des feuilles de latanier 
collectées pour recouvrir les toitures. Les seconds rassemblent les bienfaits non matériels proposés par les écosystèmes (aménités, loisirs...) et ils renvoient alors aux activités éducatives, de détente, touristiques ou bien encore religieuses pratiquées, par exemple, dans les forêts. La biodiversité peut, quant à elle, renvoyer aux services dits de support (qui incluent aussi la formation des sols, les cycles biogéochimiques); ils permettent aux trois autres catégories de services de se maintenir, les troisièmes étant, selon la terminologie du MEA, les services de régulation (rôle dans la qualité de l'air, le climat, en l'occurrence l'atténuation du réchauffement climatique, l'érosion des sols), moins étudiés ici.

2 L'objectif de cette contribution, fondée sur des recherches doctorales (Robert, 2011), est d'identifier les dynamiques qui ont affecté les paysages arborés - ceux qui sont marqués par la présence de l'arbre, qu'il soit spontané ou planté - et d'analyser les conséquences induites en matière de biodiversité et de services écosystémiques. Il s'agit ainsi d'interroger la place de l'arbre dans les paysages, d'en saisir les évolutions, en les mettant en relation avec celles qui concernent la biodiversité spécifique. Au-delà, il s'agit de déterminer quelles sont les conséquences de ces évolutions pour les sociétés montagnardes : ne sont-elles perceptibles qu'en matière de ressources exploitées ? N'y a-t-il pas aussi des conséquences d'un point de vue social et culturel? L'étude se focalise sur la région montagneuse de la province de Thừa Thiên Huế (Centre Việt Nam), où les populations, appartenant aux ethnies minoritaires, sont intimement liées aux forêts, plus largement aux formations végétales. Ici, nous considérons ces dernières comme des "sylvosystèmes ", tels que définis par Gérard Houzard (1985), à savoir une " coproduction de la nature et de la société [...] s'inscrivant dans l'espace et dans le temps ", chacun de ces "sylvosystèmes » étant «lui-même un ensemble ordonné et fonctionnel d'unités élémentaires, les sylvofaciès » (Amat, Dorize et Le Chœur, 2008). Le sylvosystème peut être considéré comme «la forêt des géographes" (ibid.) et représente ainsi «un état des paysages et des milieux à un moment donné » (Alexandre et Génin, 2012) - par analogie, nous parlerons aussi d'agrosystème pour ce qui est des "écosystèmes " agricoles. Pour connaître ces sylvosystèmes et leurs évolutions, la méthodologie adoptée mêle approche endogène et approche exogène, en vue de prendre en compte à la fois ce qui relève des processus biophysiques et ce qui relève des actions et des représentations des sociétés. Ainsi, la démarche s'intéresse-telle aux interrelations nature/société, en s'interrogeant surtout sur les rapports entretenus par les sociétés montagnardes avec les paysages arborés qui les entourent et notamment avec l'arbre, quelle que soit la composante paysagère dans laquelle il se trouve. Tout d'abord, les modalités de cette approche à la fois endogène et exogène seront précisées, avant de faire état des résultats obtenus en deux temps, l'hypothèse de départ étant fondée sur l'existence d'une évolution majeure entre l'avant-guerre et l'après-guerre - sans que la guerre en soit la seule cause (Robert, 2011). Nous nous intéresserons donc à la place occupée par l'arbre dans les paysages et aux services associés de la période précoloniale jusqu'à la guerre, puis de 1975 à aujourd'hui. Les résultats seront ensuite discutés, en interrogeant le lien entre arbre et diversité végétale, ainsi que la variation des paysages arborés et des services associés, dans le temps et dans l'espace. 


\section{Terrain, sources et méthodes}

3 La région montagneuse de Thừa Thiên Huế a été choisie comme zone d'étude afin d'identifier les changements qui ont affecté les paysages arborés des ethnies minoritaires. $\mathrm{Y}$ ont été conduites des recherches visant à acquérir une connaissance à la fois endogène et exogène des paysages. Cette distinction est reprise de la thèse de Ghislaine Marie Mellac (2000), qui, souhaitant «apporter une lecture différente de l'évolution et des facteurs de transformation du couvert végétal », emprunte deux approches : dans l'une, « les activités humaines et leurs empreintes sont abordées sans que la "parole" ne soit donnée aux hommes ", alors que l'autre "s'intéresse, par le biais d'enquêtes rétrospectives, à la signification sociale de l'organisation du couvert ». Dans le cadre de cet article, nous proposons de croiser ces deux approches, en analysant l'évolution de la place de l'arbre dans les paysages de manière distancée, en interrogeant sa fréquence et la diversité de ses espèces - approche exogène - mais tout en s'intéressant aux représentations qu'en ont les ethnies minoritaires, par la mobilisation du concept de paysage, et surtout aux services que ces dernières en tirent - approche endogène. Le concept de services écosystémiques présente l'intérêt, dans le cas d'une approche endogène, d'être anthropocentré, ce qui habituellement lui vaut plutôt des critiques. Mais on peut y voir un apport dans la réflexion sur les interrelations entre les sociétés et les paysages, en considérant ce concept comme une « approche renouvelée des questions environnementales » (Maris, préface de l'ouvrage d'Arnauld de Sartre, Castro, Dufour et Oszwald, 2014). Il peut, en effet, être un moyen "de détruire le mythe persistant de la séparation entre l'homme et la nature, et de consciemment réintégrer les hommes dans la nature, au cœur de la biodiversité dont ils font partie » (Loreau, 2016). L'usage du terme de sylvosystème (voir supra), réintégrant l'homme, et la définition retenue de la biodiversité - "résultat d'une coévolution naturelle et sociale» (Simon, 2006) - procèdent de la même logique. Par ailleurs, la mobilisation du concept de services écosystémiques permet ici de compléter l'approche en matière de dynamiques paysagères, en considérant ce que les écosystèmes apportent aux sociétés, au-delà de ce qui serait perceptible par l'analyse des dynamiques d'occupation des sols. En effet, sont ici considérés les services d'approvisionnement mais aussi ceux culturels qui n'ont a priori pas d'impacts visibles sur les paysages ou seulement indirectement - en influençant l'exploitation forestière (exemple des forêts sacrées: voir infra). La méthodologie et les sources mobilisées s'attachent donc à considérer les caractéristiques des paysages, en insistant ici sur leurs composantes arborées, autant que les points de vue des populations qui les peuplent et les services qu'elles en tirent, ce que nous verrons après une présentation du terrain d'étude, justifiant son choix.

\section{La région montagneuse de Thừa Thiên Huế}

4 Le choix de la province de Thừa Thiên Huế s'explique notamment parce qu'elle est l'une de celles qui ont été les plus affectées par la guerre (Robert, 2016). Aux nombreux bombardements, y compris au napalm, se sont en effet ajoutés des épandages d'herbicides. Ils ont été particulièrement intenses dans la province qui était alors rattachée au Sud-Việt Nam (figure 1). Ses montagnes, surtout à hauteur de la vallée d'A 
Lưới, ont constitué une cible privilégiée (ibid.). Elles ont ainsi connu de profonds bouleversements au cours de cette période.

Figure 1. Épandages d'herbicides au Sud-Việt Nam (1965-1971)

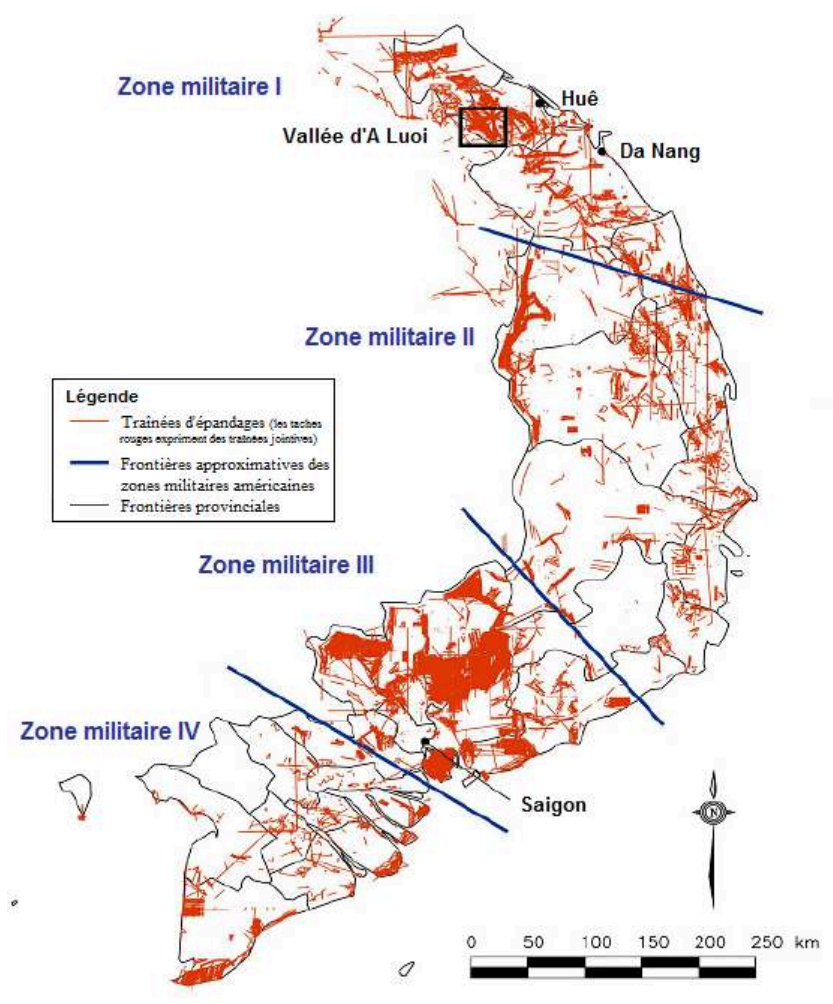

Source : Hatfield Consultants LTD et du Comité 10-80, d'après les données du département de l'Armée des États-Unis, http://www.hatfieldgroup.com/UserFiles/File/ContaminantMonitoringAgentOrange/ VietNamHighlights/SprayLines.PDF, légende traduite par l'auteur.

5 La région montagneuse s'étend à l'ouest et au sud de la province de Thừa Thiên Huế (figure 2). Elle est marquée par la prédominance de sylvosystèmes spontanés et constitue ainsi l'unité paysagère la plus forestière de la province (figure 3). S'y concentrent les ethnies minoritaires, absentes des collines et de la plaine qui sont les domaines de l'ethnie majoritaire kinh. Ce sont, par ordre d'importance, les Pakô (ou Pa Cô), les Katu, les Tà Ôi, les Pahy et les Vân Kiều, tous du groupe môn-khmer (Đặng Nghiêm Vạn et al., 2000). Les premiers se concentrent, comme les Tà Ôi, presque exclusivement dans le district occidental d'A Lưới, qui regroupe près de $75 \%$ des individus appartenant aux ethnies minoritaires de la province (SNNVPTNT, 2002). Ces populations étaient installées sur ce territoire bien avant la période coloniale (1885-1954, voir infra). Elles y pratiquaient l'agriculture itinérante sur brûlis. Mais quelle place occupait l'arbre dans les paysages de ces montagnards? Pour le déterminer, les recherches conduites ont visé à acquérir, comme souligné plus haut, une connaissance à la fois endogène et exogène des paysages. 
Figure 2. Relief dans la province de Thừa Thiên Huế

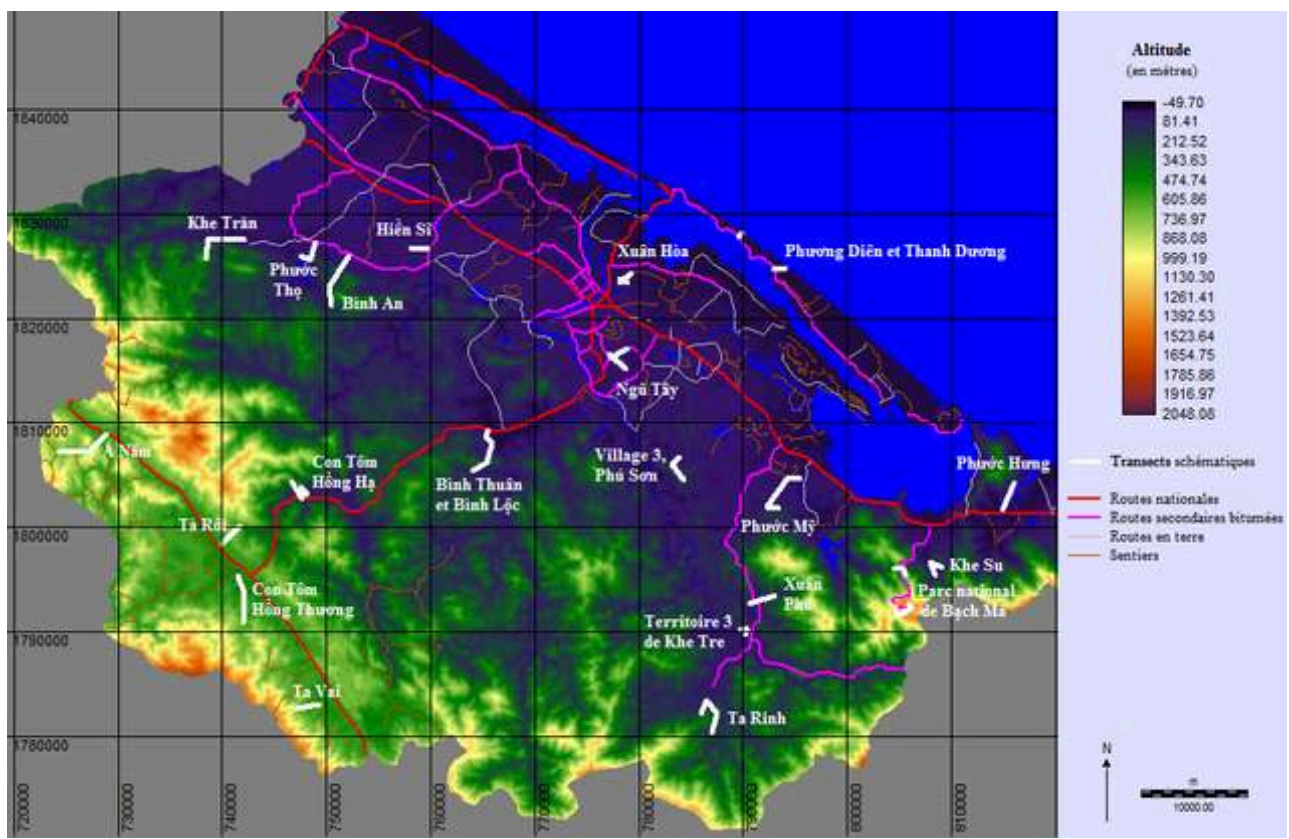

Géoréférencement : UTM 48N/WGS 84.

Sources : d'après la carte topographique NE-48-16 (Huê), réalisée en 2001 à l'échelle 1/250 000 par I'Office cartographique de l'état-major général de l'armée populaire du Viêt Nam (d'après la carte à l'échelle 1/250 000, UTM, de 1982, modifiée d'après la carte à l'échelle 1/100 000, UTM, de 1986 et la carte de l'aviation à l'échelle 1/500 000 de 1994); courbes de niveau interpolées à partir du modèle TIN (Triangulated Irregular Netwok) parabolique, sans contrainte.

Figure 3. Occupation des sols en 2003 dans la province de Thừa Thiên Huế

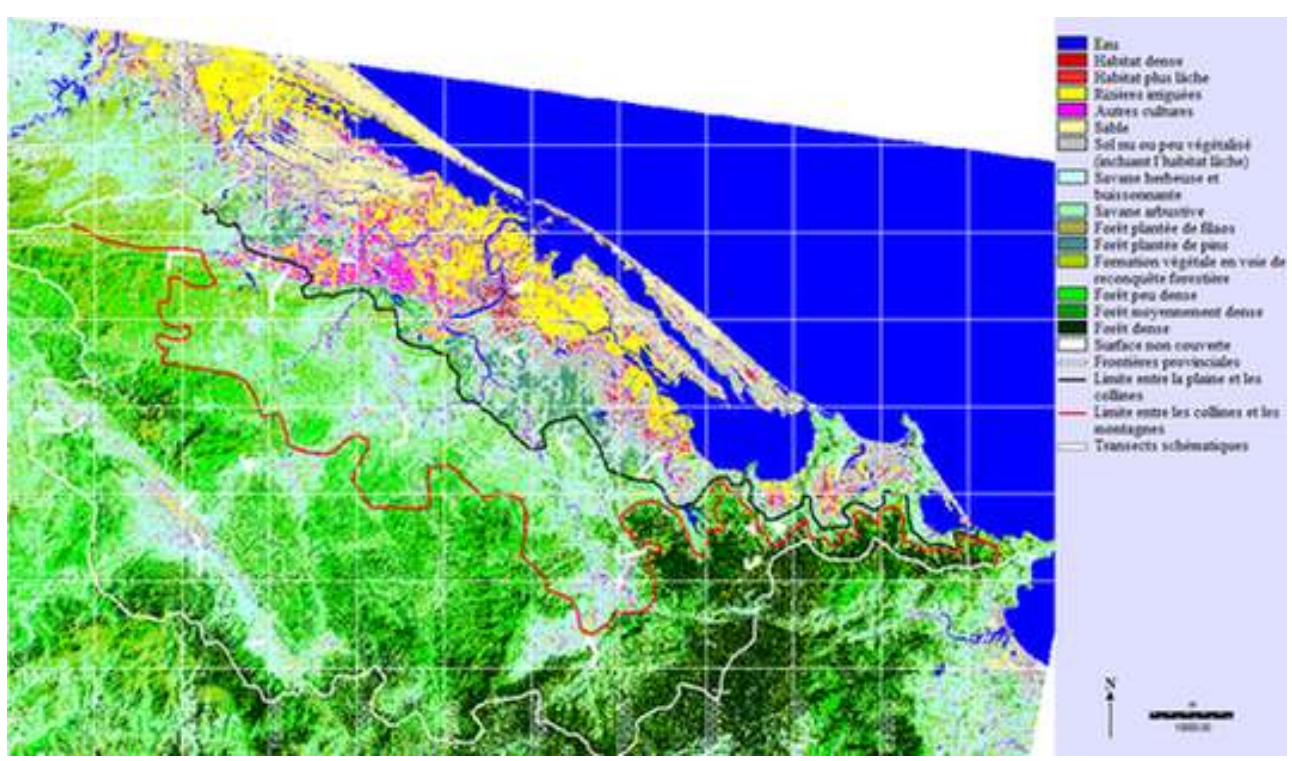

Géoréférencement : UTM 48N/WGS 84.

Sources: d'après les images satellitales Landsat 7 ETM + du 31 janvier et du 21 avril 2003 (traitées avec Idrisi Kilimanjaro). 


\section{Sources et méthodes, pour une connaissance endogène et exogène des paysages}

6 Afin de connaître les pratiques, les usages et les paysages tels qu'ils sont perçus par les montagnards, des entretiens semi-directifs ont été menés dans sept villages. Plusieurs critères ont prévalu dans le choix de ces derniers : ils sont répartis dans l'ensemble de la région montagneuse ; ils ont été plus ou moins affectés par les pratiques militaires au cours de la guerre (Robert, 2011) et ils sont majoritairement peuplés d'ethnies minoritaires. Ce sont les villages (figure 2) de :

- Khe Trăn, peuplé de Pahy, situé dans le district de Phong Điền au nord ;

- Tà Rôi, dont les habitants appartiennent à l'ethnie Tà Ôi, situé dans le district d'A Lưới - le seul qui est exclusivement montagneux - à l'ouest ;

• À Năm, Con Tôm Hồng Hạ, Con Tôm Hồng Thương et Ta Vai, peuplés tous les quatre principalement par des Pakô et situés dans le district d'A Lưới ;

- Ta Rinh, village katu du district de Phú Lộc, au sud.

Dans ces villages, entre trois et cinq habitants ont été interrogés, en privilégiant des personnes âgées pour obtenir des informations sur la situation passée. Pour compléter cette source majeure, ont aussi été consultées des données d'archive (en France et au Việt Nam) et la littérature existante, qui renseigne notamment sur la période coloniale et celle de la guerre.

8 Parallèlement, la connaissance des paysages arborés est aussi passée par la réalisation de cartes d'occupation des sols, à des dates-clés, choisies en fonction des temporalités de la société vietnamienne (figure 4). Plusieurs périodes ont ainsi été identifiées au cours de l'histoire contemporaine, depuis le XIx siècle, étant entendu qu'à chaque période correspondent des pratiques propres susceptibles de marquer les paysages. En 1885, la conquête française met fin à la période précoloniale et ouvre une nouvelle ère, celle de la colonisation. Cette dernière s'achève avec la guerre d'Indochine, dite de décolonisation: en 1954, les accords de Genève séparent le pays en deux zones distinctes, le Nord communiste et le Sud, où se situe Thừa Thiên (nom alors donné à la province), placé sous la protection des États-Unis. Mais le conflit demeure latent et très vite, en 1962, une nouvelle guerre éclate, celle du Việt Nam, opposant les NordVietnamiens aux Sud-Vietnamiens, soutenus par l'armée américaine. La guerre prend fin en 1975 ; le pays est réunifié et la collectivisation s'impose au Sud-Việt Nam jusqu'en 1986. À cette date, débute la période actuelle, dite du "Renouveau», avec la libéralisation de l'économie (passage à l'économie de marché à orientation socialiste) et l'ouverture du pays. Plusieurs dates marquent ainsi des changements, séparant ces périodes; des états des lieux devaient y être dressés et comparés entre eux, afin d'identifier les dynamiques paysagères et de connaître notamment l'évolution de la place de l'arbre dans l'occupation des sols. L'objectif était d'acquérir une connaissance exogène des paysages arborés, de manière objective, en considérant non plus les représentations, la manière dont ils sont perçus, mais leur matérialité. Pour dresser les cartes d'occupation des sols, des sources primaires iconographiques ont été privilégiées. Elles se sont toutefois révélées lacunaires à mesure qu'on remontait le passé et le nombre de dates retenues pour dresser des états des lieux des paysages a dû être réduit :

- Pour l'état des lieux en 2003-2007, deux images satellitales Landsat datant de 2003 ont été sélectionnées, à deux saisons distinctes, afin de faciliter l'identification des formations 
végétales, en considérant les différences phénologiques des formations végétales. La première date de la fin de la saison des pluies ( 31 janvier) et offre ainsi de plus forts contrastes que la seconde, prise au cours de la saison sèche (21 avril). Parallèlement, pour valider les classes d'occupation des sols identifiées et compléter notre connaissance de ces dernières, des relevés de terrain (2006-2007) ont été réalisés le long de transects, qui ont traversé les villages dans lesquels ont été conduits les entretiens auprès des montagnards (figure 2).

- 1975 marque la fin de la guerre, plus exactement des guerres : d'Indochine (1945-1954, de décolonisation, contre les Français) puis du Việt Nam (contre les Américains), même si est surtout considérée ici la seconde, qui a eu le plus d'impacts sur les paysages des montagnes de Thừa Thiên Huế. Pour cet état des lieux, seule une image satellitale, aux résolutions spectrale et spatiale plus faibles que celles des images de 2003, a pu être acquise; elle date de la saison sèche (14 mars 1975). Elle a été complétée par les entretiens menés auprès des villageois, notamment pour remplacer les relevés de terrain dans la validation des catégories d'occupation des sols identifiées.

Figure 4. Séquences chronologiques : sources et temporalités

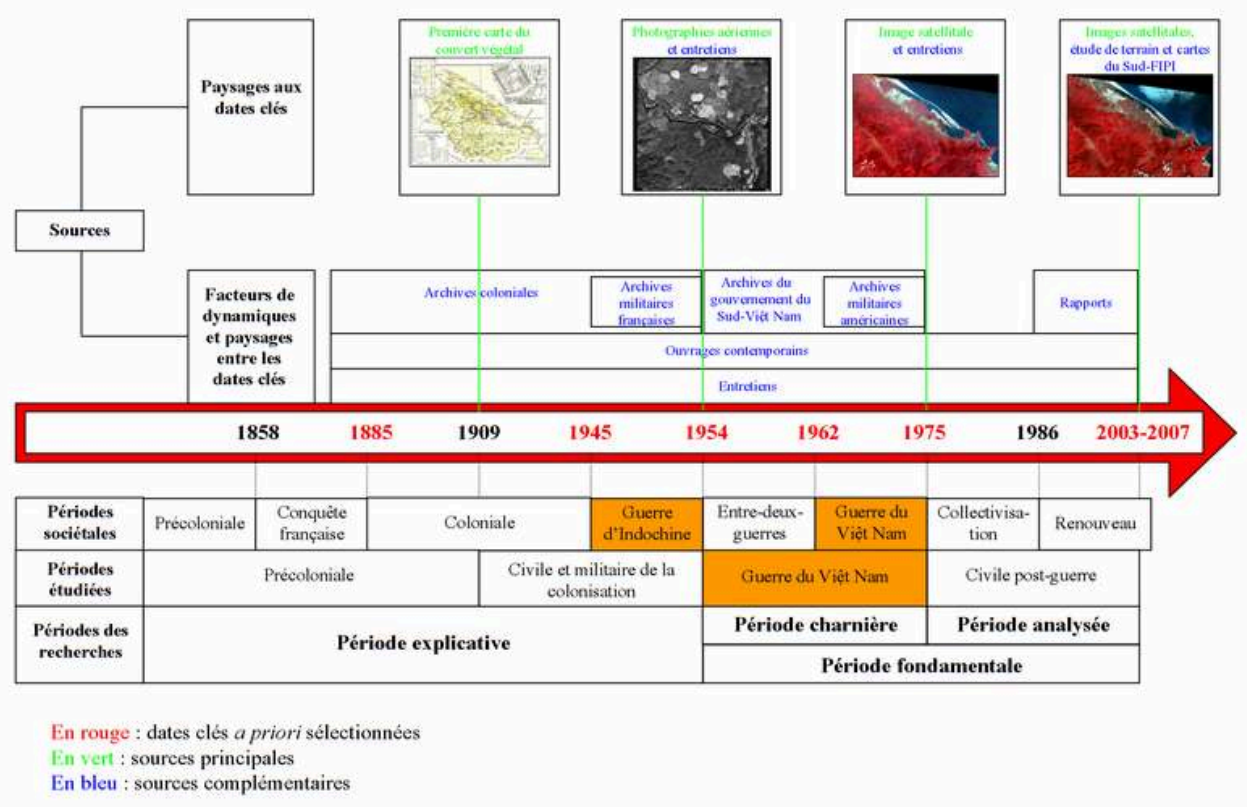

Source : Robert, 2011.

- 1954 est la seule date précédant la guerre du Việt Nam pour laquelle des données ont pu être obtenues afin de connaître l'occupation des sols. Il s'agit de photographies aériennes qui présentent l'avantage d'avoir une plus grande résolution spatiale que les images satellitales Landsat mais nécessitent un temps de traitement plus long. Par conséquent, les cartes n'ont été dressées qu'à hauteur de certains transects. Ces photographies datent plus exactement de 1952-1954 et ont été acquises en France, auprès du département de l'armée de l'air du service historique de la Défense.

- Avant 1954, la seule source disponible est une carte de la végétation réalisée au début du ${ }_{\mathrm{xx}}^{\mathrm{e}}$ siècle à l'échelle de la province de Thừa Thiên par Antoine de Chabert-Ostland et Lucien Gallois et disponible dans leur Atlas général de l'Indochine française (1909). Elle renseigne sur les paysages au début de la colonisation. 
Quatre états des lieux de l'occupation des sols ont ainsi finalement été dressés, en se fondant sur des sources principales mais qui ont dû être complétées par d'autres (figure 4). L'identification des dynamiques est passée par une analyse diachronique. Les états des lieux ont été confrontés deux à deux dans un système d'information géographique (SIG) - hormis 1909 car, sur la source correspondante, les informations, notamment les localisations, sont trop imprécises -, ce qui a permis de connaître les permanences et les mutations au cours de chacune des périodes. Ces dernières ne possèdent pas toutes le même poids au regard de la recherche menée. La période 1954-1975, correspondant à la guerre du Việt Nam, est considérée comme charnière (figure 4) car elle est à l'origine d'importants changements (Robert, 2011), nous le verrons. La période qui suit est celle de l'analyse puisqu'il s'agit d'y étudier la manière dont les changements se manifestent dans les paysages, surtout dans les actuels, dans la mesure où la recherche relève de la biogéographie historique - les paysages du passé sont convoqués pour comprendre les paysages actuels. La période de 1954 à aujourd'hui est ainsi considérée comme fondamentale au regard de la recherche, alors que celle antérieure n'est étudiée que pour déterminer quels étaient les paysages avant le changement majeur que constitue la guerre ; par ailleurs, son analyse ne peut se fonder que sur des sources lacunaires et la connaissance des dynamiques survenues au cours de cette période n'est alors que partielle.

10 Les résultats de l'analyse des dynamiques de l'occupation des sols ont ensuite été mis en relation avec les témoignages des villageois; là apparaît la richesse de l'approche paysagère: "Compte tenu des relations entre les pratiques, les "perceptions" et les mutations du paysage, l'analyse des représentations en général et des images véhiculées en particulier est importante pour comprendre l'évolution des paysages. " (Servain-Courant et Yengué, 2011.) Et, comme souligné, pour comprendre les rapports entre les paysages arborés des montagnes de Thừa Thiên Huế et les ethnies minoritaires qui les peuplent, est aussi mobilisé le concept de «services écosystémiques »: à partir des informations collectées lors des entretiens, il s'agit d'identifier les apports de l'arbre et des écosystèmes qu'il forme pour ces sociétés, en matière de ressources (services d'approvisionnement) mais aussi du point de vue culturel (services culturels). De l'analyse ainsi conduite, il ressort une évolution quant à la place de l'arbre dans les paysages; quelles en sont les incidences en ce qui concerne les services rendus aux populations? De la colonisation - voire avant - jusqu'à la guerre, l'arbre spontané apparaît comme un élément majeur des paysages. Quels services rendait-il aux ethnies minoritaires?

\section{L'arbre spontané, élément majeur des paysages de la colonisation à la guerre}

11 La carte la plus ancienne dont nous disposons est celle de Antoine de Chabert-Ostland et Lucien Gallois ( 1909 ; figure. 5), qui constitue la première carte de la végétation de la province de Thừa Thiên mais elle n'apporte que des informations lacunaires. Les forêts n'y sont en effet distinguées qu'en fonction des ressources qu'elles offrent, à savoir essences ou bois. On considère que les forêts fournissant des essences sont denses; or, en 1909, elles sont omniprésentes dans la région montagneuse de Thừa Thiên. Pour les autres forêts présentées sur la carte (figure 5), qui offrent du bois, on suppose qu'elles sont moins denses et elles se concentrent dans les montagnes les plus accessibles 
depuis la plaine - où les densités de population ainsi que la pression exercée sur les sylvosystèmes sont plus fortes -, notamment au nord. Les témoignages collectés auprès des montagnards confirment cette omniprésence supposée des forêts denses. Pour autant, ils doivent être considérés avec précaution. En effet, les sylvosystèmes sont indistinctement perçus comme des forêts, y compris les savanes arbustives. C'est ce qui ressort de la confrontation entre ces témoignages et les cartes d'occupation des sols réalisées à partir des photographies aériennes prises entre 1952 et 1954. Celles-ci révèlent en effet que, dans les vallées principales - celle d'A Lưới surtout (à l'est-nordest de la carte, figure 6) - comme dans les secondaires, les savanes sont largement présentes et se dessine, à proximité des villages notamment, comme le long du transect d'A Năm pris en exemple ici, un paysage en mosaïque. Celui-ci s'explique par la présence de diverses formations végétales, des sylvofaciès à des stades de reconquête différents. Ceci est à mettre en lien avec l'agriculture itinérante sur brûlis que pratiquent à cette époque les montagnards (Gourou, 1940 ; Condominas, 2003) : le stade varie selon le temps depuis lequel la parcelle cultivée a été abandonnée. Toujours est-il que, malgré cette diversité, la présence de l'arbre spontané est majeure à cette époque.

Figure 5. Carte de la végétation dans la province de Thừa Thiên en 1909

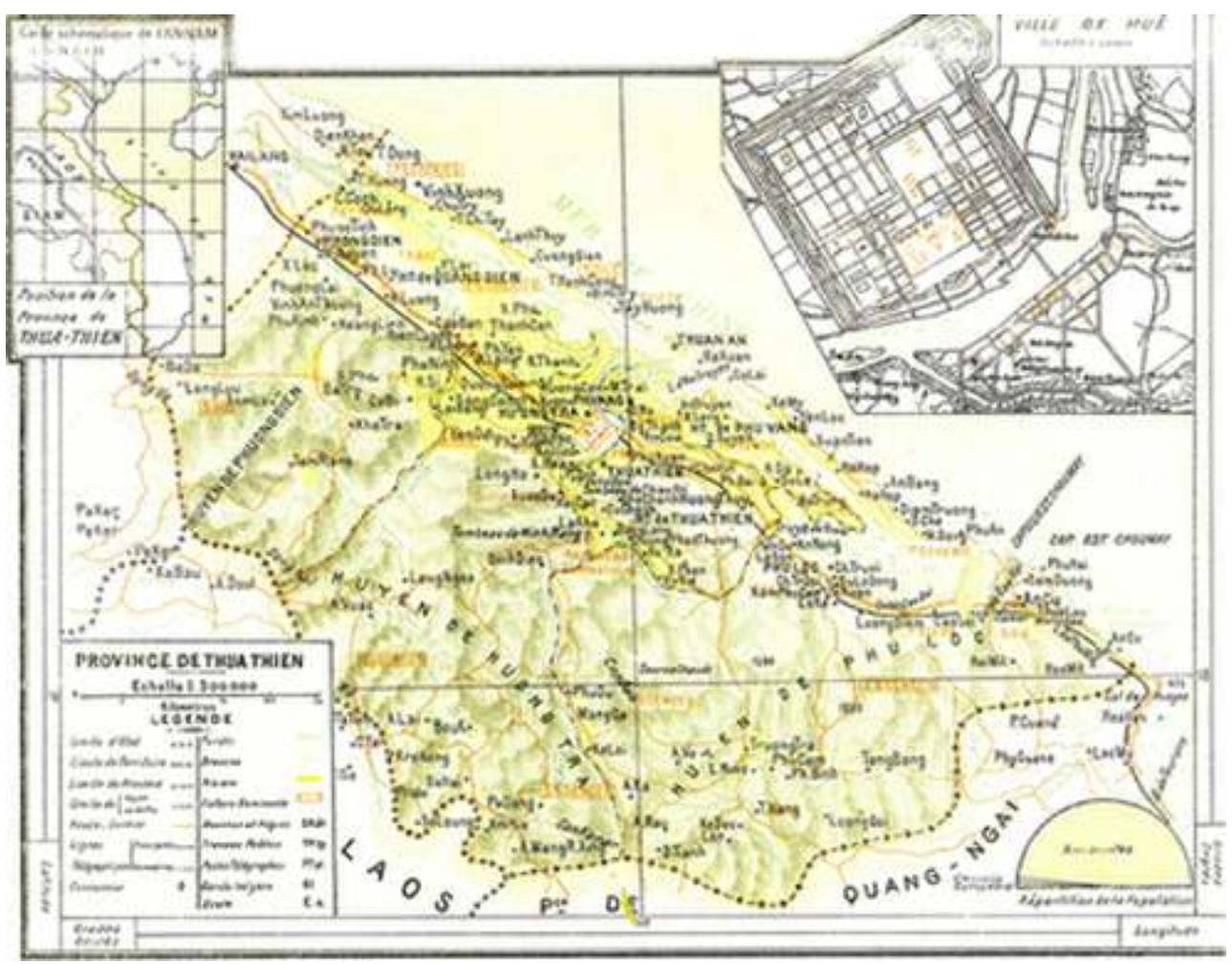

Source : Antoine de Chabert-Ostland et Lucien Gallois, 1909, Atlas général de I'Indochine française, Hanoi-Haiphong, Imprimerie d'Extrême-Orient, 1909, 169 cartes et plans.

Les montagnes de Thừa Thiên appartiennent au domaine tropical humide et, à ce titre, les forêts s'y caractérisent par une diversité végétale importante (Bergonzini et Lanly, 2000 ; Huynh Thu Ba, 2003 ; Nguyễn Nghĩa Thìn et Mai Văn Phô, 2003). Cette diversité caractérise autant les espèces présentes en sous-bois que celles qui atteignent les étages supérieurs. Elles sont ombrophiles, sciaphiles en sous-étage mais héliophiles dans la strate supérieure. Certaines sont considérées comme précieuses, recherchées 
pour la dureté de leur bois : Erythrophleum fordii, Hopea pierrei, Sindora siamensis, Tarrietia javanica... D'autres sont de moindre valeur: Parashorea stellata (Dipterocarpaceae), Madhuca pasquieri (Sapotaceae), Michelia spp., Pometia pinnata, Paranephelium spirei (Sapindaceae), Aquilaria crassna (Thymelaeaceae), Dysoxylum spp. (Meliaceae), Prunus arborea (Rosaceae), certaines de la famille des fagacées... Les villageois interrogés attestent la présence à cette époque de ces diverses espèces ligneuses. Ils précisent que les grands arbres sont nombreux, atteignant 1-1,2 $\mathrm{m}$ de diamètre - les montagnards estiment plus facilement les diamètres des arbres que leurs hauteurs -, aux côtés d'autres, de taille moyenne, à savoir 20-30 cm de diamètre. S'ajoutent en sous-étage d'autres ligneux, des fougères, des lataniers... La présence de sylvosystèmes moins évolués concourt aussi à la diversité, puisqu'on y retrouve d'autres espèces ligneuses encore, davantage héliophiles.

Figure 6. Occupation des sols en 1954 le long du transect d'A Năm

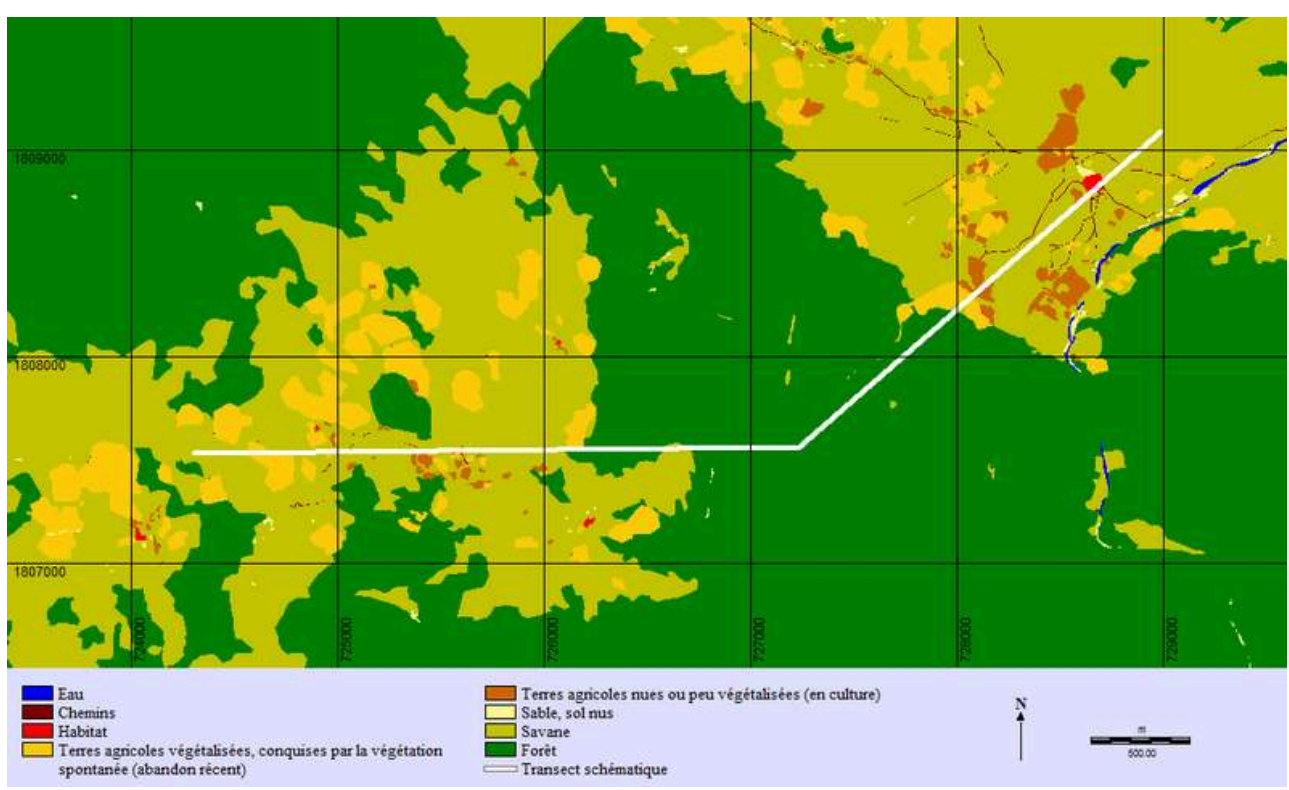

Géoréférencement : UTM 48N/WGS 84.

Sources: d'après les photographies aériennes du service historique de la Défense - département de l'Armée de l'air (SHD-DAA), plot 765, mission TV 125 du 1er mars 1954 (1/20 000-25 000).

Qu'ils soient forestiers ou savanicoles, ces sylvosystèmes, omniprésents dans les montagnes de Thừa Thiên, répondent de façon complémentaire aux besoins des ethnies minoritaires en bois et en produits forestiers non ligneux. Ils rendent ainsi des services dits d'approvisionnement, selon la typologie souvent reprise, définie par le MEA (2005) : comme souligné, ces derniers incluent l'ensemble des ressources fournies par les écosystèmes, utilisables par l'homme (nourriture, matériaux de construction...). Concernant le bois, les montagnards l'exploitent pour la cuisson des aliments et pour la construction notamment de leurs habitations, dans des lieux de collecte distincts. Le bois de chauffe est souvent coupé dans les savanes proches des villages et (ou) sur les futures parcelles agricoles, lors des déboisements précédant la mise en culture. Le bois d'œuvre est, lui, exploité dans les forêts denses. Des produits forestiers non ligneux sont aussi prélevés dans ces dernières : feuilles de rotin ou de latanier pour recouvrir les toits, tubercules pour l'alimentation... En effet, comme le souligne Pierre Gourou (1940), « les forêts procurent aux montagnards mille ressources, elles leur donnent des 
matériaux pour leurs maisons et leur outillage, des denrées alimentaires, du combustible, des produits tinctoriaux ».

L'arbre spontané fait aussi partie intégrante du paysage agricole. Les montagnards pratiquent en effet l'agriculture itinérante sur brûlis où sylvosystèmes et agrosystèmes sont intimement liés. Des parcelles couvertes de forêts sont déboisées pour être mises en culture : la végétation est coupée puis incendiée, afin que la cendre fertilise le sol. Elles sont cultivées pendant un à trois ans, selon la qualité de ce dernier. Puis elles sont abandonnées, pendant une période de jachère variable - selon les situations, selon les sources aussi (Gourou, 1940 ; Condominas, 2003) $)^{3}$ : la forêt recolonise alors les parcelles, du moins la dynamique de reconquête est-elle enclenchée. Chaque année, de nouvelles forêts sont ainsi déboisées, dans les limites des territoires villageois, pour être cultivées. En devenant de manière temporaire des agrosystèmes ${ }^{4}$, ces sylvosystèmes offrent ainsi des ressources alimentaires. L'arbre demeure par ailleurs présent sur les parcelles, y compris pendant la période de culture. En effet, bien souvent, les plus grands arbres ne sont pas abattus - les montagnards n'ont pas les outils adéquats pour cela, selon leurs déclarations - et s'ils le sont, de nombreux troncs demeurent dispersés sur les parcelles, ce dont attestent des photographies de l'époque coloniale, disponibles au Centre des archives d'outre-mer (CAOM).

L'arbre spontané est aussi au centre de croyances. Les écosystèmes qu'il forme rendent alors des services dits culturels, rassemblant les bienfaits non matériels fournis par les écosystèmes (aménités, loisirs...). Croyant en l'esprit de la forêt, les ethnies minoritaires accordent le statut de forêts sacrées à certaines. Ces forêts sont celles qui abritent cet esprit et elles deviennent alors des lieux de prière, notamment pour les Pahy. Ce sont aussi des forêts qui sont utilisées comme cimetières, pour les Pahy, Tà Ôi et Pakô (Huynh Thu Ba, 2003). Dans ces forêts sacrées, tout prélèvement est souvent proscrit, de sorte que les services culturels influencent ici les services d'approvisionnement rendus par les sylvosystèmes. Au-delà, les paysages arborés constituent le cadre de vie de ces populations : elles vivent « dans la forêt », selon leurs déclarations. Même si les villages sont en fait plutôt entourés de savanes, du moins dans les vallées (figure 6), les arbres spontanés n'en sont pas moins présents à proximité immédiate des habitations. Leur importance pour les montagnards est telle qu'il n'est pas rare que les végétaux ligneux donnent leur nom aux villages : «Une ethnie de telle région a une façon particulière de nommer le village par le nom des arbres et des hommes. Par exemple, si le village où on habite a beaucoup d'iry (banians, appelés iry dans la langue de l'ethnie), ce village est appelé iry. Et quand on entend le nom du village, on sait bien ce qui le caractérise et on comprend que c'est un village de Tà Ôi. Ou bien, les Pa Cô habitent une région qui a beaucoup d'along (rhododendrons dans la langue de l'ethnie), on la dénommera Pa Cô Along. " (Nguyễn Khánh Phong, enseignant au lycée d'A Lưới, cité par Nguyễn Thu Hương, 2013).

L'arbre et les écosystèmes qu'il forme sont ainsi partie intégrante des paysages des ethnies minoritaires, prenant une place centrale dans l'occupation des sols autant que dans les pratiques, les usages, les croyances (Gourou, 1940 ; Huynh Thu Ba, 2003) et plus largement dans la culture de ces populations. Avec la guerre, ils revêtent aussi un caractère protecteur, de refuge, dissimulant les montagnards à la vue des soldats américains. Ces derniers dirigent alors leurs attaques contre cette composante arborée des paysages. 
les bombardements, les épandages d'herbicides sont nombreux dans la province (voir supra) et ils affectent particulièrement la région montagneuse (Robert, 2011 et 2016 ; figure 7). À travers ces pratiques militaires, l'objectif des soldats américains et sud-vietnamiens est surtout de détruire le couvert foliaire sous lequel les Việt-cộng se dissimulent. Comme ceux-ci trouvent refuge dans les montagnes, les forêts de cette région constituent une cible majeure. Il en résulte des bouleversements perceptibles dans les paysages. Les sylvosystèmes régressent et certaines forêts sont remplacées par des savanes (figure 8). Pour autant, la dynamique est aussi progressive, dans les espaces délaissés par les montagnards à cause des combats (Robert, 2011 et 2016).

Après-guerre, «le recul de la forêt "- selon le titre de l'ouvrage de Rodolphe De Koninck (1997) - se poursuit, s'accélère même, et les paysages arborés des ethnies minoritaires se transforment, faisant évoluer avec eux les services rendus par les sylvosystèmes.

Figure 7. Épandages militaires d'herbicides dans la province de Thừa Thiên

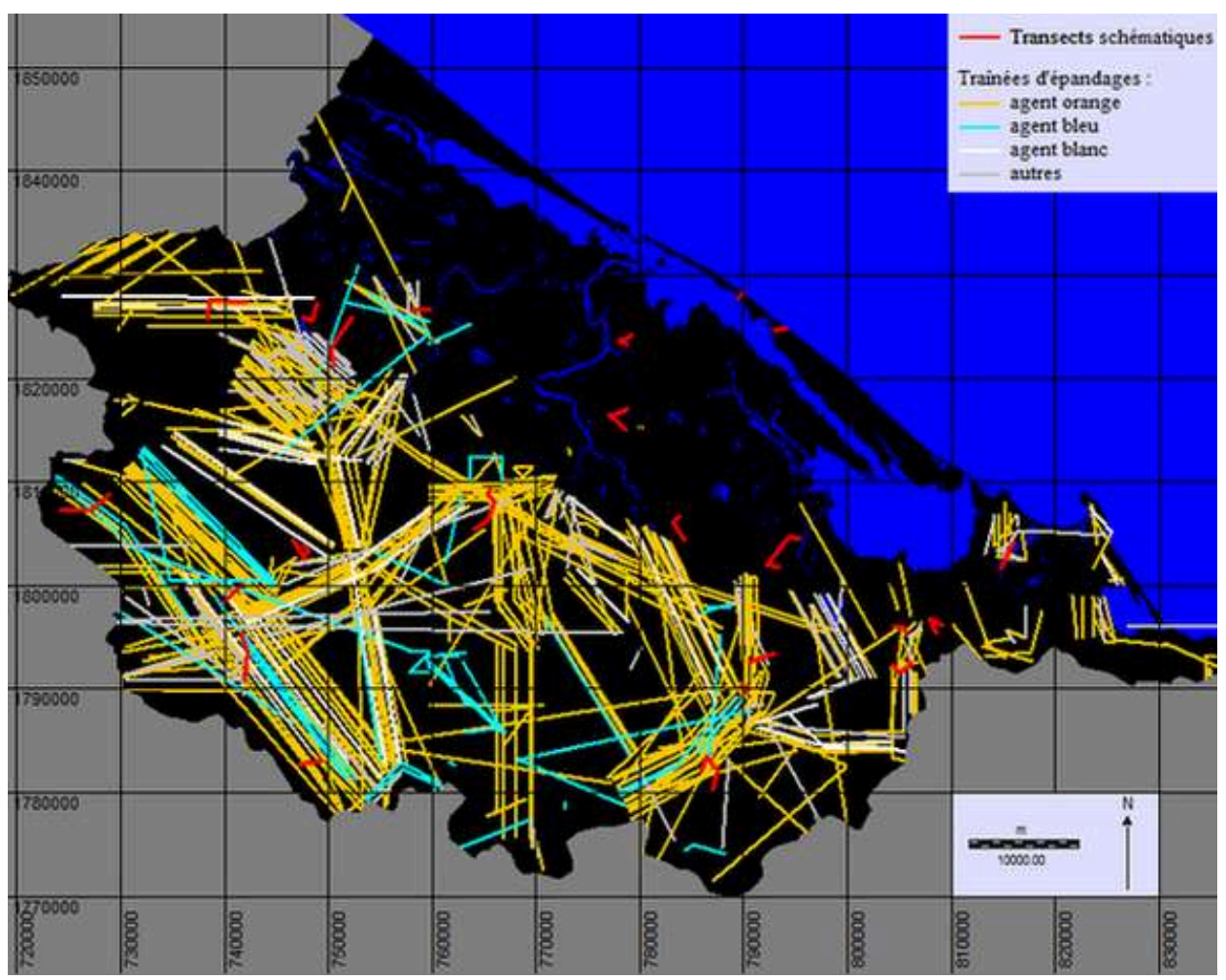

Géoréférencement : UTM 48N/WGS 84.

Source: d'après la carte des épandages d'herbicides du Forest Inventory and Planning Institute (FIPI). 
Figure 8. Dynamiques de l'occupation des sols entre 1954 et 1975 le long du transect d'A Năm

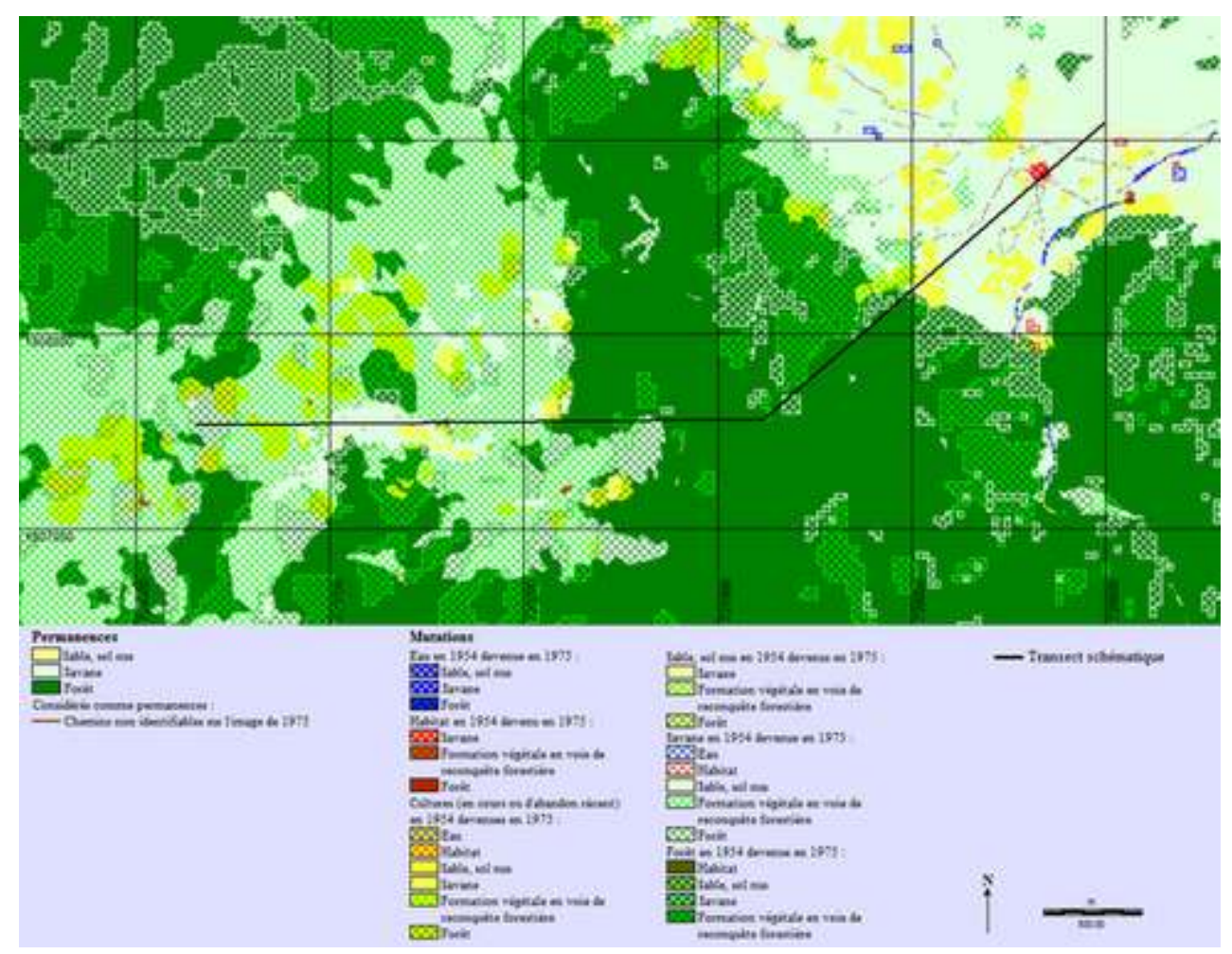

Géoréférencement : UTM 48N/WGS 84.

Sources : d'après les photographies aériennes du SHD-DAA, plot 765, mission TV 125 du 1 er mars 1954, 1/20 000-25 000) et l'image Landsat 2 MSS du 14 mars 1975.

\section{Après-guerre, de l'arbre spontané à l'arbre planté}

Après-guerre, les paysages des montagnards changent progressivement. Dès le lendemain du conflit, la plupart des ethnies minoritaires sont sédentarisées - les dernières le seront au début des années 1990 (cas de Ta Vai, en 1992). Elles sont alors établies dans les vallées principales, plus accessibles - donc plus facilement contrôlables -, celles-là mêmes dont les ligneux ont été affectés par les attaques américaines. Pour les montagnards qui résidaient ailleurs, la sédentarisation est synonyme de déplacement et elle ajoute alors aux effets de la guerre dans la transformation des paysages des montagnards. La présence de l'arbre s'amoindrit. Pour tous, la sédentarisation s'accompagne aussi d'un changement de pratiques agricoles. Les ethnies minoritaires, qui ne pratiquaient jusque-là que l'agriculture itinérante sur brûlis, adoptent la riziculture irriguée, qui n'était pratiquée que par les Kinh - ethnie majoritaire. Dans ces agrosystèmes permanents, qui se concentrent dans les vallées (photo 1), l'arbre n'a plus sa place. Et, pour les ethnies minoritaires, ce changement implique l'apprentissage de nouvelles techniques culturales (photos $2 \mathrm{~A}$ et B). Les anciennes pratiques ne sont pas abandonnées pour autant mais les montagnards se voient contraints de les adapter, à la demande du gouvernement et en raison de la réduction des territoires villageois (liée à la sédentarisation). L'agriculture sur brûlis continue à être pratiquée sur les versants (photo 1) mais l'itinérance est abandonnée et le temps de jachère est alors raccourci à trois ans. Les montagnards pratiquent ainsi ce mode de culture en alternance sur deux champs seulement. Dans ces agrosystèmes 
aussi, la présence de l'arbre s'amoindrit, ne demeurant présent que sur les rares parcelles laissées plus longtemps en jachère (photos 3 A et B) - contre l'avis des autorités. De manière générale, l'arbre spontané ne fait plus partie des systèmes culturaux, du moins sa place s'amenuise-t-elle.

Photo 1. Pratique de l'agriculture sur brûlis sur les versants, et de la riziculture irriguée dans la vallée

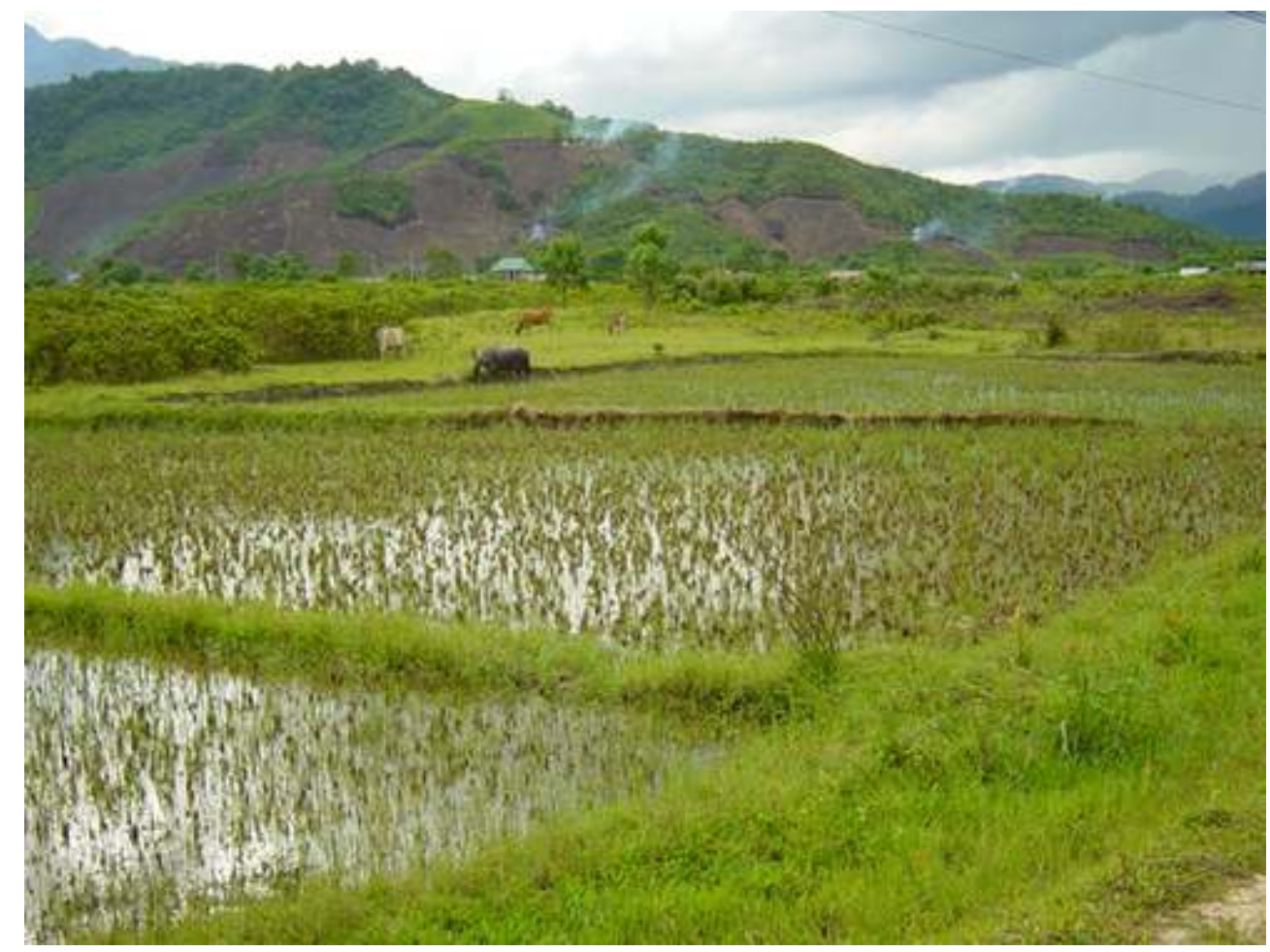

Exemple dans le village de Ta Vai.

Source : Amélie Robert, 2007. 
Photos 2a. La riziculture irriguée, adoption de nouvelles techniques culturales par les montagnards

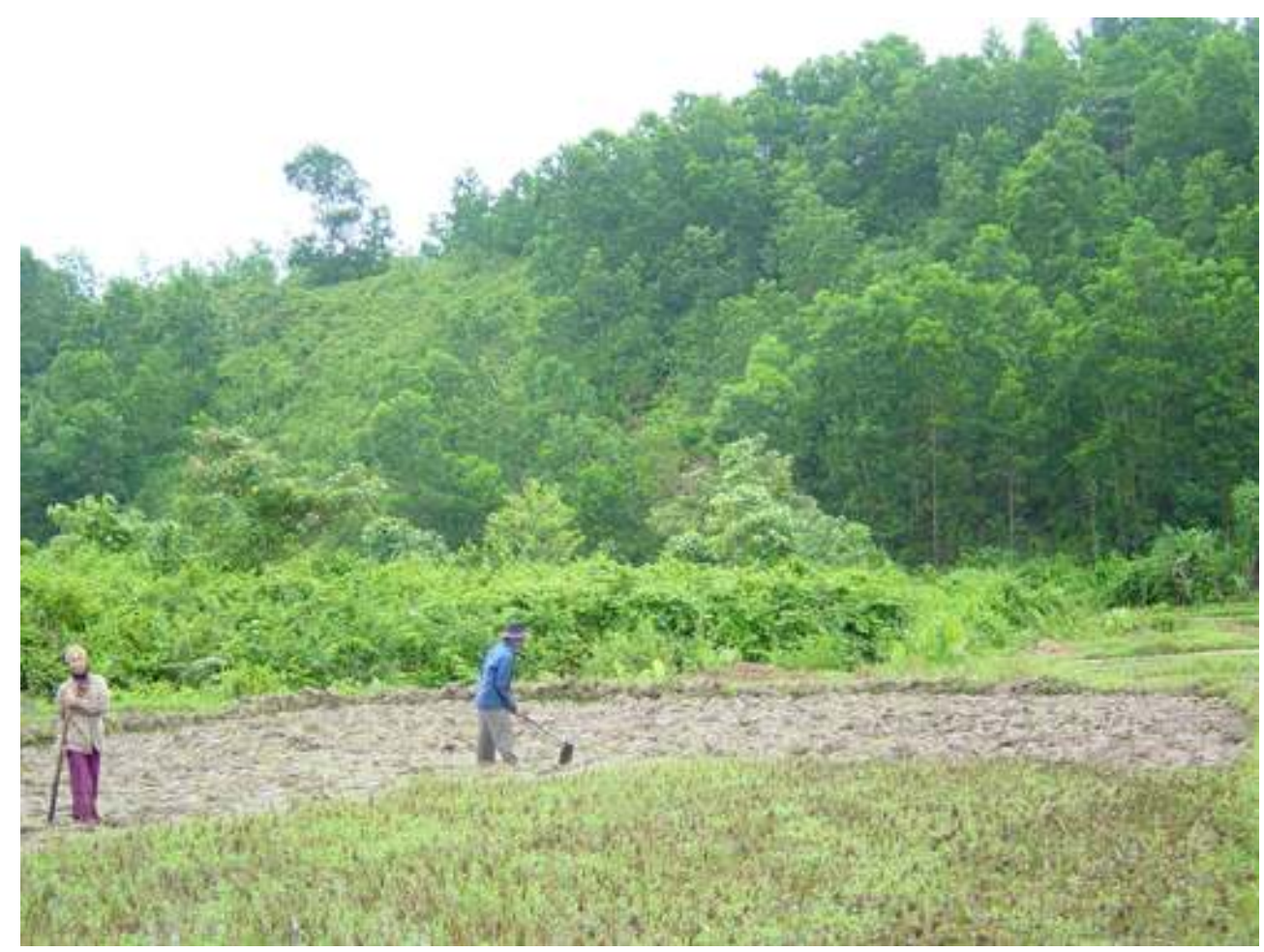

Préparation du sol avant inondation. Exemple dans le village de Ta Rinh.

Source : Amélie Robert, 2007 
Photos $2 \mathrm{~b}$. La riziculture irriguée, adoption de nouvelles techniques culturales par les montagnards

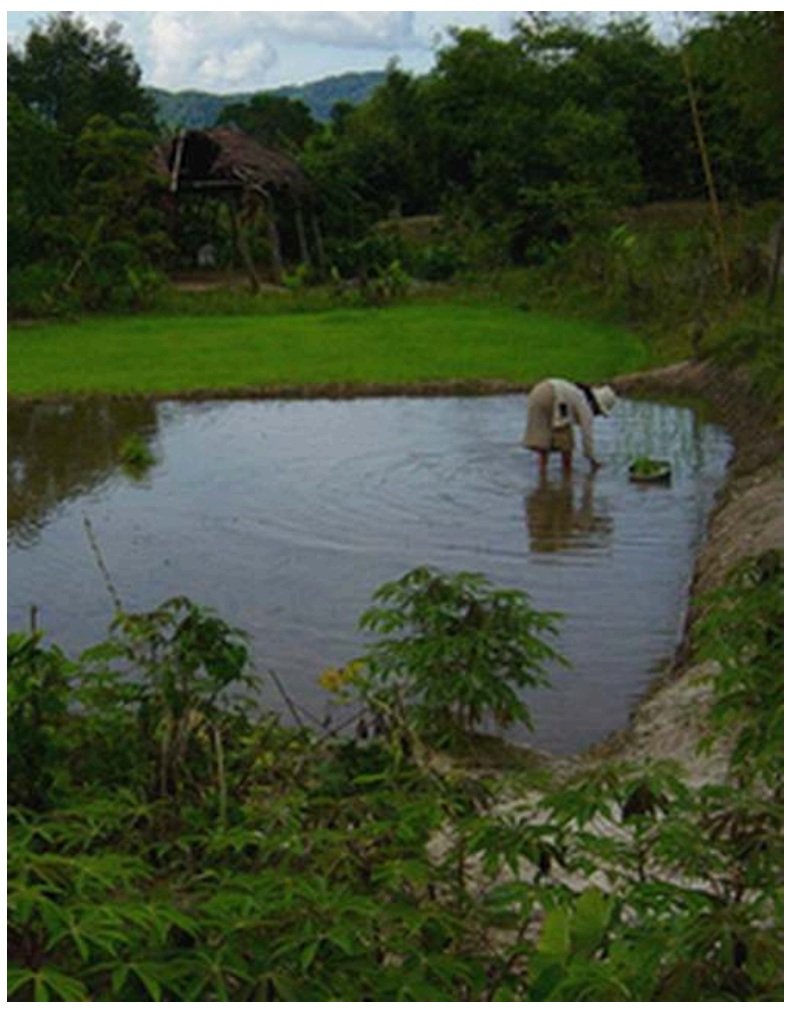

Repiquage du riz. Exemple dans le village de Ta Rinh.

Source : Amélie Robert, 2006. 
Photos 3a. Une moindre présence de l'arbre spontané sur les parcelles où est pratiquée la riziculture sèche

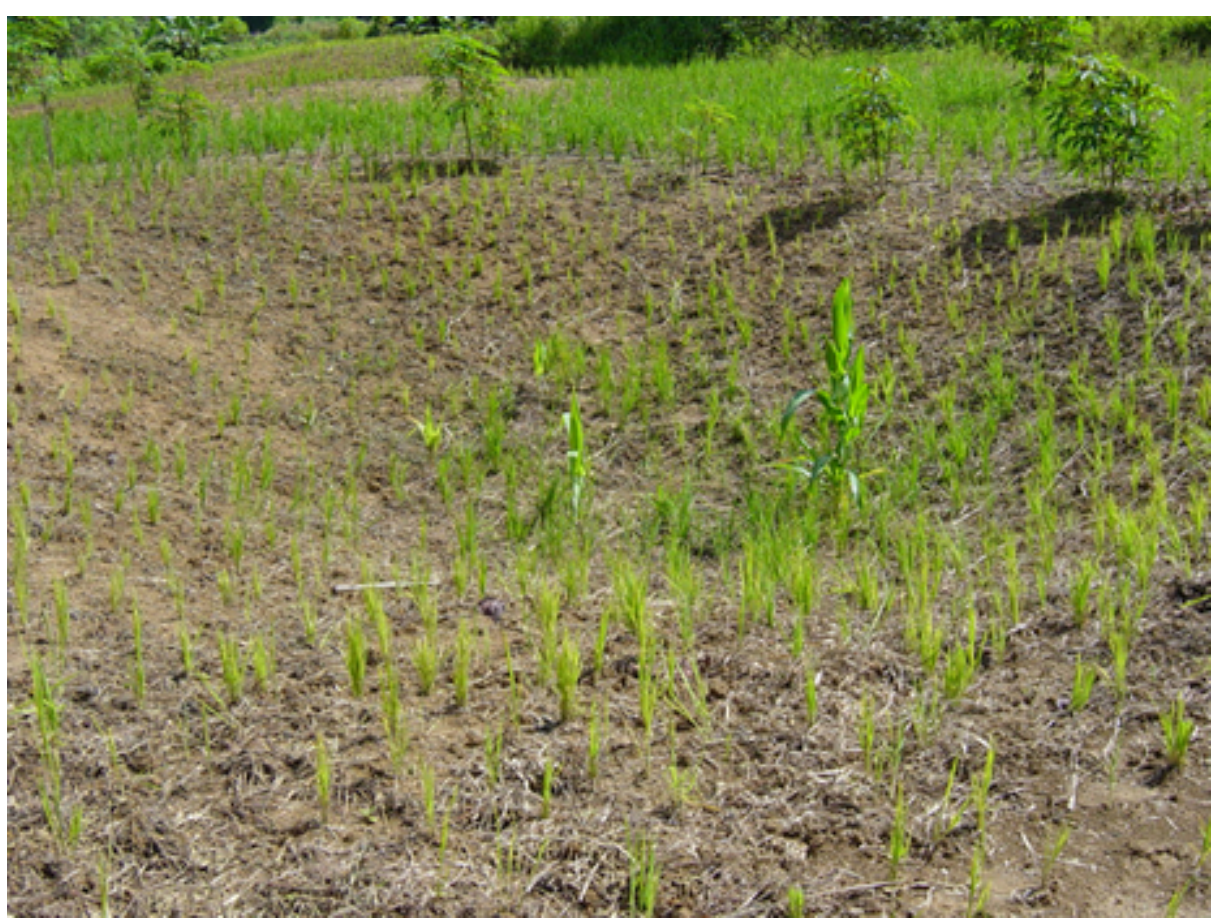

Dans la vallée : absence de l'arbre spontané. Exemple dans le village d'A Năm. Cette parcelle est en fait cultivée en permanence.

Source : Amélie Robert, 2006.

Photos $3 \mathrm{~b}$. Une moindre présence de l'arbre spontané sur les parcelles où est pratiquée la riziculture sèche

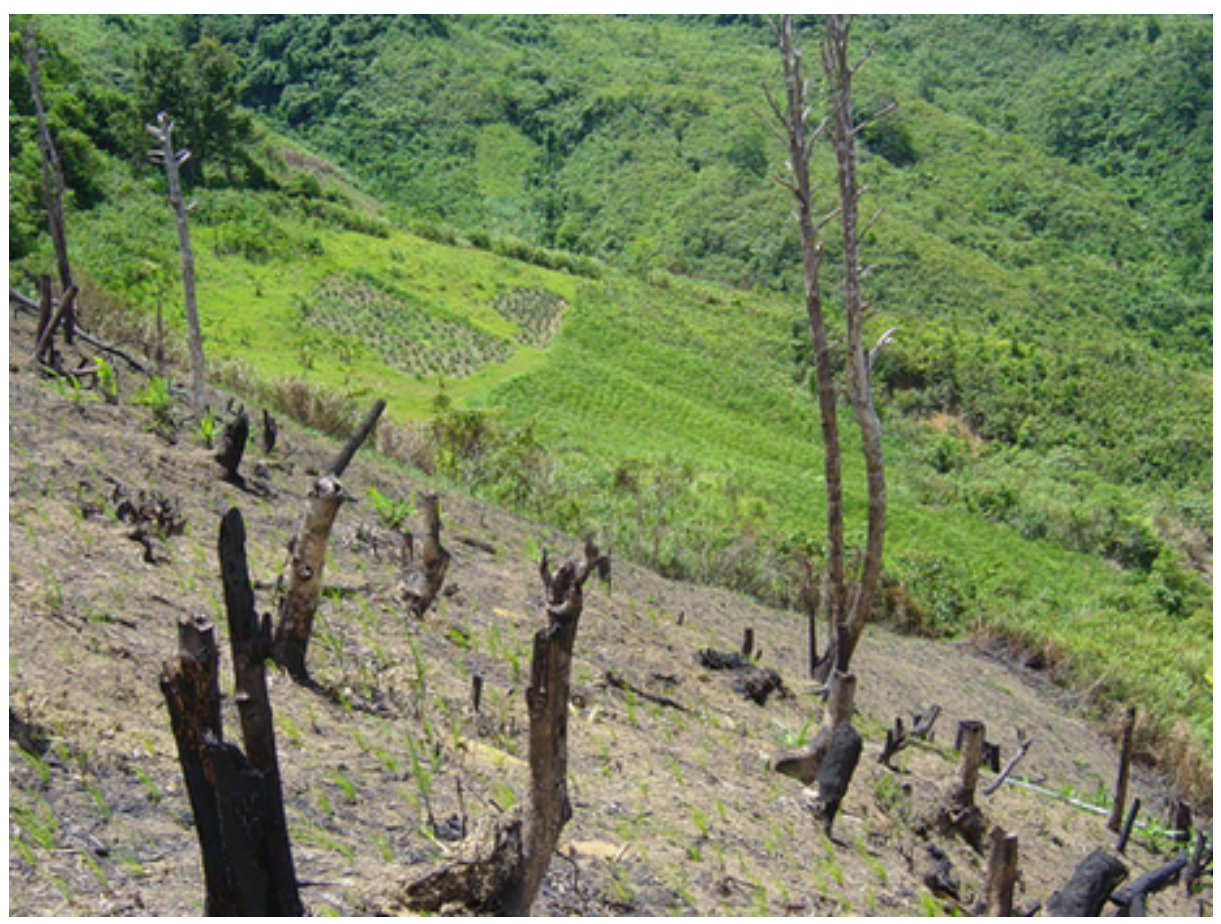

Sur un versant : présence de troncs témoignant d'un temps de jachère plus long, contre l'avis des autorités.

Source : Amélie Robert, 2006. 
La disparition de l'arbre spontané est d'autant plus importante que les changements paysagers se poursuivent. Le " recul de la forêt » s'accélère après-guerre (Robert, 2011 et 2016), comme on peut le percevoir à hauteur du transect d'A Năm, ici pris en exemple (figure 9). Les causes sont à la fois l'intensification de l'exploitation forestière et la progression de l'agriculture sédentaire. Ces deux évolutions s'expliquent par la sédentarisation des ethnies minoritaires mais aussi par l'arrivée de colons kinh qui, originaires de la plaine, viennent s'installer dans des nouvelles zones économiques établies dans les collines et les montagnes, souvent à la demande des autorités. La population des montagnes augmente et se concentre sur un territoire plus restreint, de sorte que la pression s'accroît sur les sylvosystèmes les plus accessibles puis bien vite sur de plus éloignés. Il s'agit de mettre en culture de nouvelles terres, de prélever aussi le bois et les ressources non forestières nécessaires. Ces prélèvements sont d'autant plus grands que, pour les ethnies minoritaires, il ne s'agit plus seulement de satisfaire leurs propres besoins. Elles vendent aussi parfois le bois qu'elles exploitent aux Kinh. La conséquence est que les sylvosystèmes denses, à forte biodiversité ligneuse, sont repoussés à des distances croissantes, au profit des savanes (figure 2). Désormais, la présence de l'arbre spontané est moindre; sa diversité est plus faible et elle profite davantage aux espèces héliophiles qui peuplent les savanes, plus étendues. Dans les vallées principales, notamment celle d'A Lưới, les rizières irriguées prédominent et l'arbre spontané a disparu (photo 4).

Figure 9. Dynamiques de l'occupation des sols entre 1975 et 2003 le long du transect d'A Năm

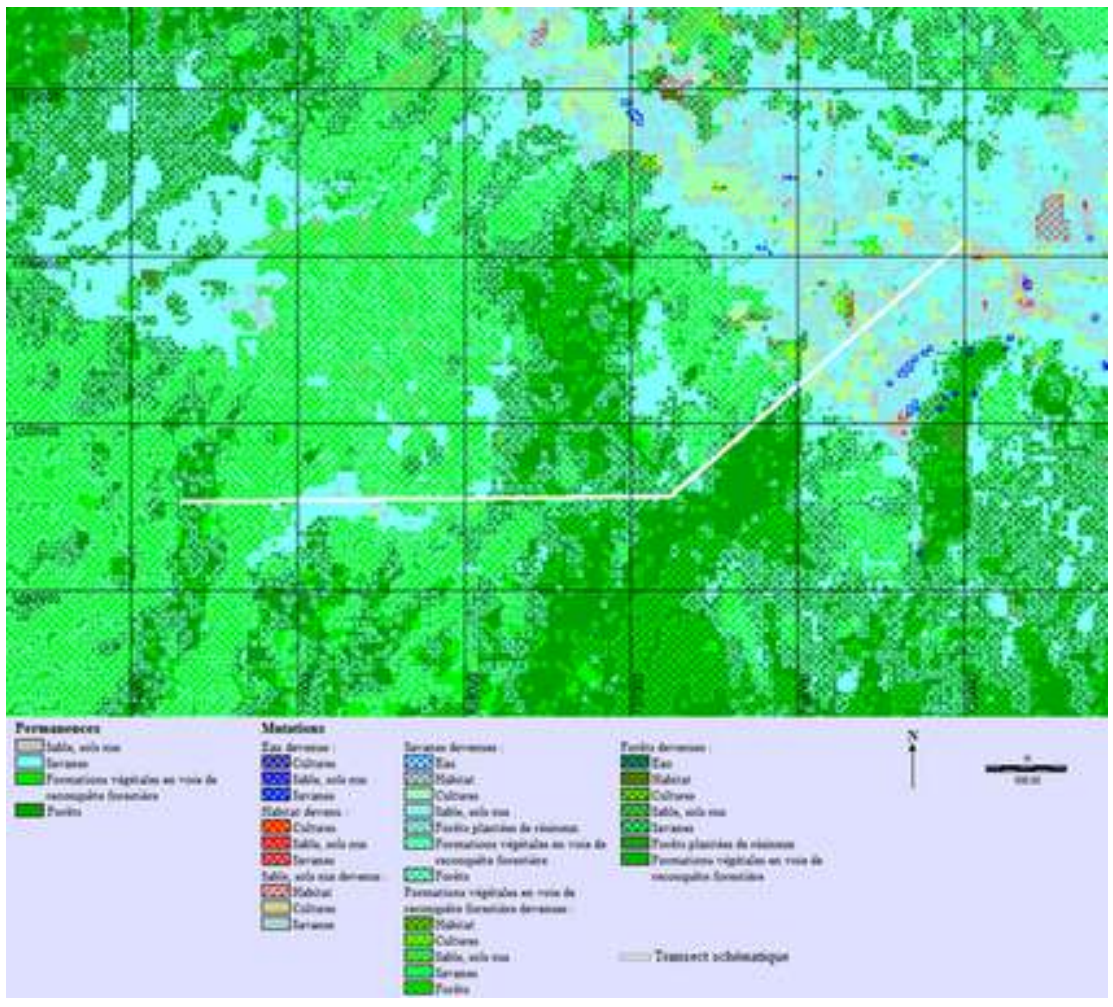

Géoréférencement : UTM 48N/WGS 84.

Sources : d'après l'image Landsat 2 MSS du 14 mars 1975 et les images Landsat 7 ETM + du 31 janvier et du 21 avril 2003. 
Photo 4. Prédominance des rizières irriguées et disparition de l'arbre spontané dans les vallées principales. Exemple de la vallée d'A Lưới

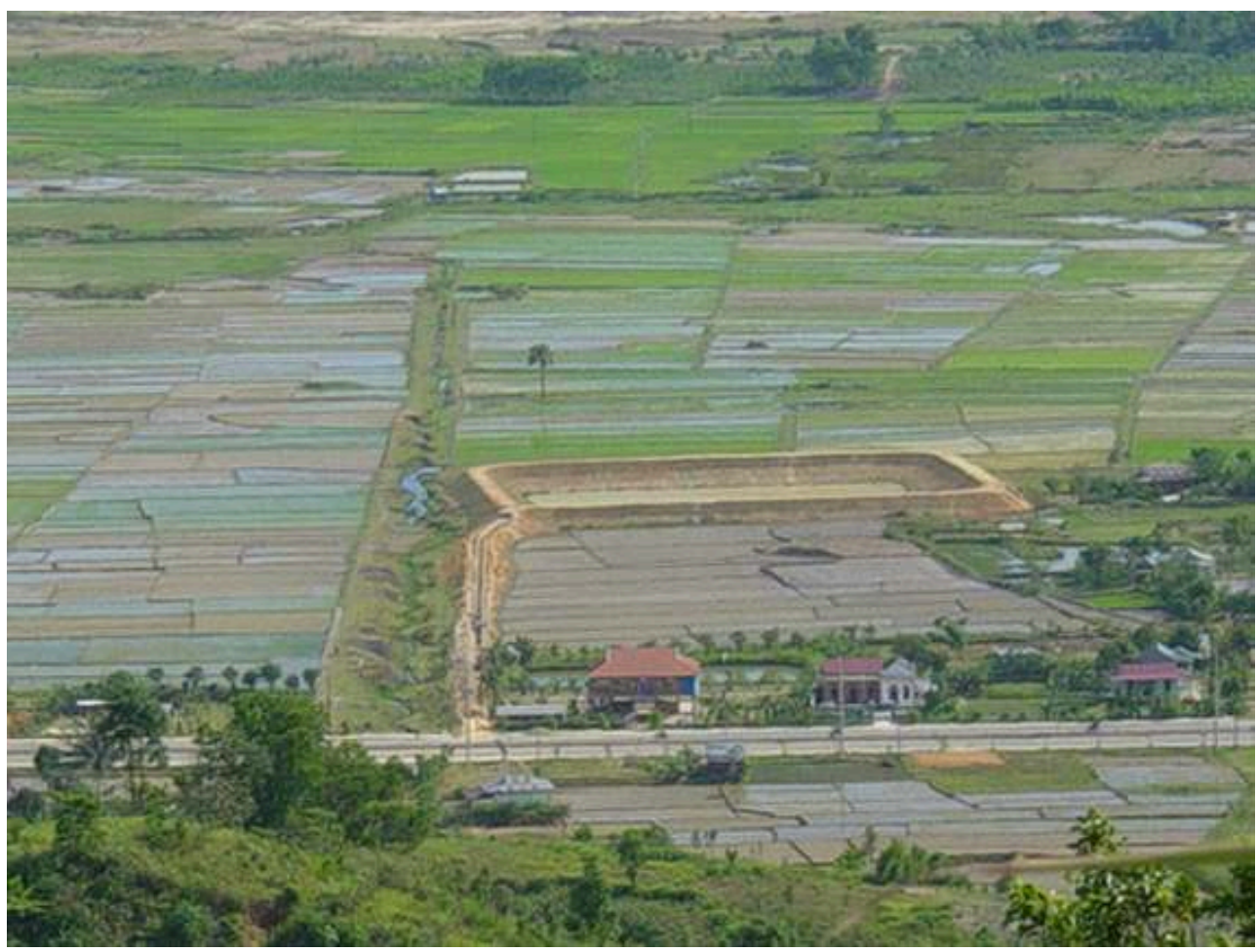

Un bassin de stockage de l'eau est d'ailleurs en cours de construction au centre de la photo, en vue de faciliter l'irrigation des rizières.

Source : Amélie Robert, 2006 (depuis le village de Tà Rôi).

Cette transformation des paysages a plusieurs conséquences pour les montagnards, en matière de services rendus par les sylvosystèmes. Concernant les services d'approvisionnement associés aux arbres, le bois est collecté à des distances croissantes. Ceci vaut d'autant plus pour le bois d'œuvre que pour le bois de chauffe qui, lui, peut être prélevé dans les savanes. Concernant les services culturels, les changements qui affectent les paysages ont des incidences aussi. Les forêts sacrées se raréfient, voire disparaissent, comme toutes les forêts. Celles qui subsistent ne sont en réalité souvent que des savanes arbustives. Le caractère sacré peut même être transféré parfois à des arbres remarquables, reliques des forêts disparues - il est intéressant de souligner que ces arbres n'appartiennent pas à des espèces dites précieuses (en l'occurrence offrant du bois d'œuvre de qualité). «La forêt primaire est largement morte et avec elle les ressources qu'en tirait la population, et tout le quadrillage des lieux symboliques avec les génies, les repères chamaniques en général ont été mis à mal ", comme le soulignent Bernard Doray et Concepcion Doray (2013). Même si ces auteurs attribuent cette disparition de la "forêt primaire" aux seules pratiques militaires américaines - ce que contredisent les sources (voir supra; Robert, 2011 et 2016) -, il n'en demeure pas moins que, effectivement, la forêt dense et, avec elle, l'arbre spontané se raréfient après-guerre, nous l'avons vu. Le cadre de vie, l'environnement des montagnards se transforment ainsi et, avec la disparition de la forêt, cadre de vie originel, ce sont les repères culturels des ethnies minoritaires qui disparaissent. 
22 L'arbre dans le paysage se raréfie; il se transforme aussi. Sa diversité est désormais moindre, limitée aux espèces héliophiles près des villages. Mais apparaissent aussi de nouveaux sujets, plantés ceux-là. En effet, depuis les années 1990, des pins, des eucalyptus, des canneliers et désormais surtout des acacias ${ }^{5}$ (Amat et al., 2010 ; Nambiar et al., 2015) forment des plantations monospécifiques et équiennes ; s'ajoutent aussi des hévéas. Ces plantations s'étendent sur les versants, près des parcelles cultivées (photos 5 A et B ; photo $2 \mathrm{~A}$ en arrière-plan). Elles côtoient parfois sur les mêmes terres des cultures annuelles (manioc notamment ; photo 6) mais qui ne sont complantées que les deux ou trois premières années. La fonction de l'arbre diffère ici par rapport à celle que remplissait l'arbre spontané : alors que celui-ci était au service des cultures annuelles (pour fertiliser les sols), l'arbre planté est voué à remplacer ces cultures l'objectif est d'optimiser l'usage du sol et d'obtenir des récoltes en attendant de pouvoir tirer profit des plantations, du moins autant que possible.

Photo 5a. Plantations s'étendant sur les versants près des villages

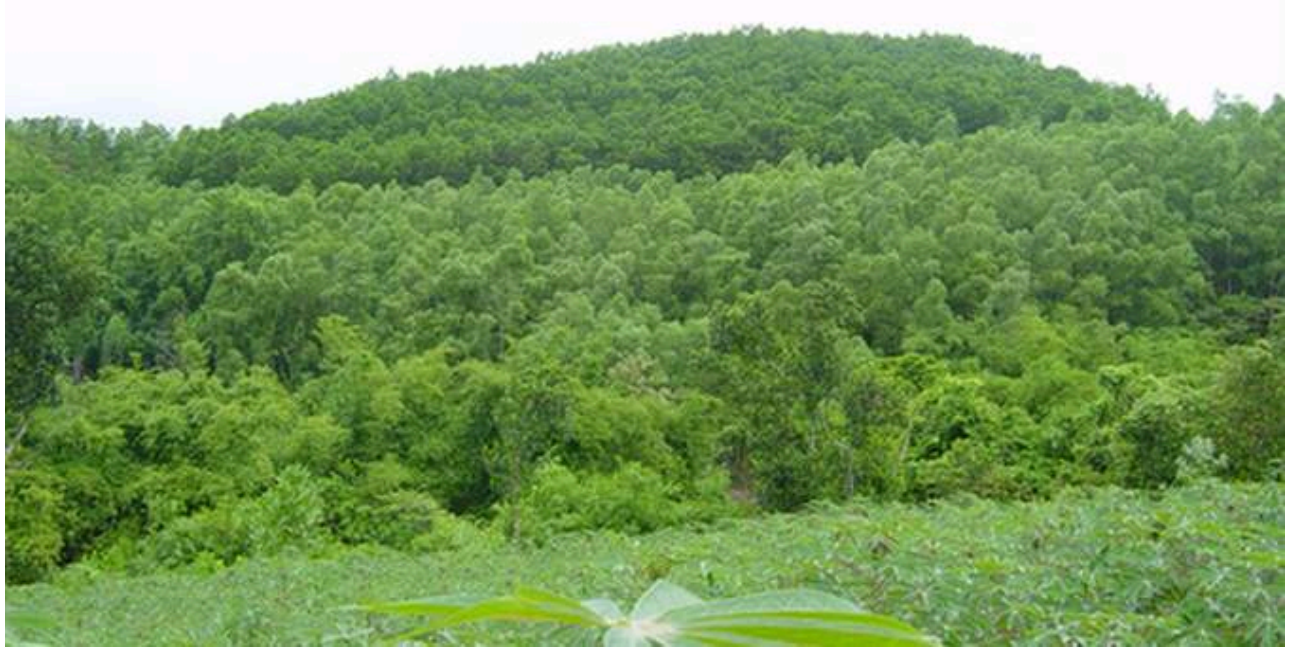

Forêts plantées d'acacias. Exemple dans le village de Con Tôm Hồng Hạ.

Source : Amélie Robert, 2006. 
Photo 5b. Plantations s'étendant sur les versants près des villages

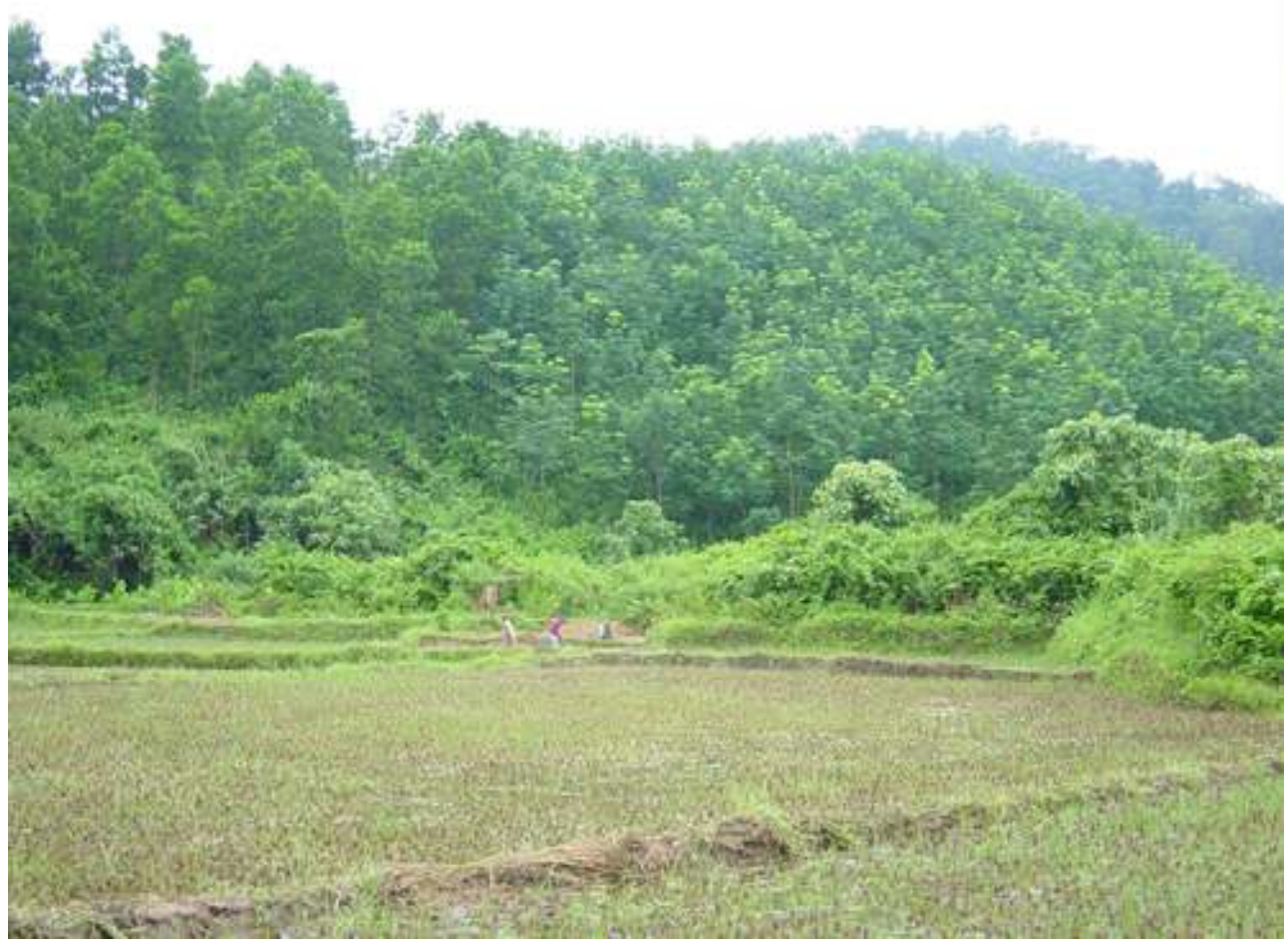

Plantations d'acacias et d'hévéas. Exemple dans le village de Ta Rinh. Source : Amélie Robert, 2007. 
Photo 6. Une plantation (agricole) d'hévéas complantée de manioc. Exemple dans le village de Con Tôm Hồng Hạ

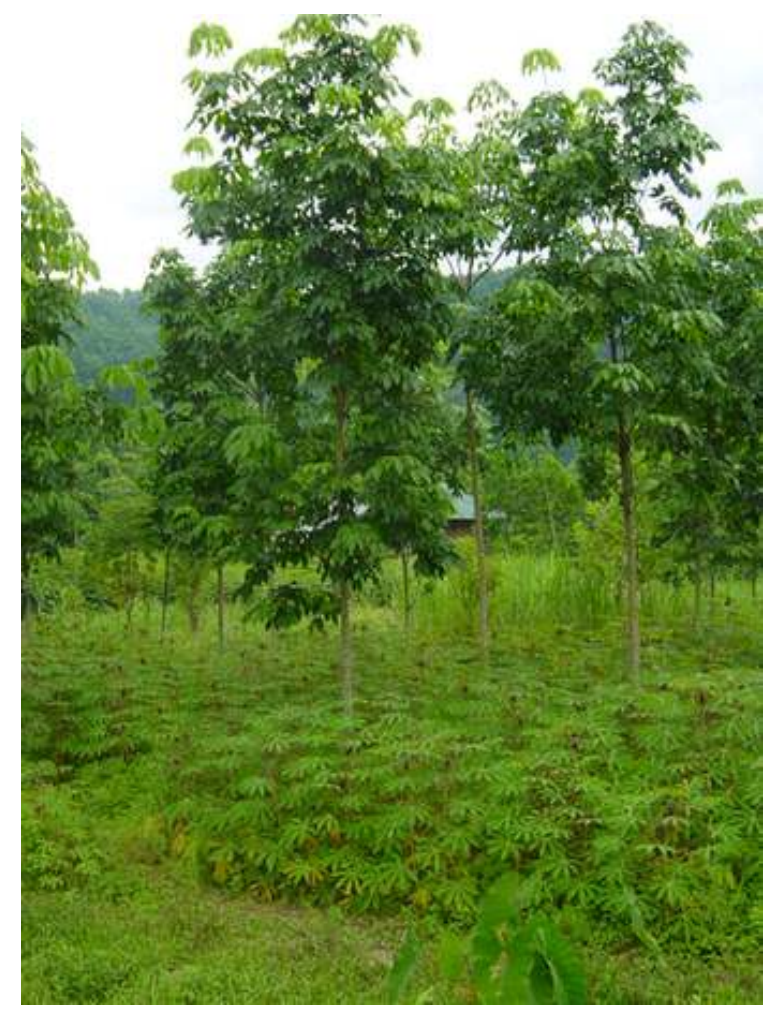

Sur certaines parcelles, ce sont des acacias qui sont complantés de manioc.

Source : Amélie Robert, 2007.

Ces plantations offrent des services écosystémiques aux ethnies minoritaires, au moins des services d'approvisionnement. Les montagnards peuvent en effet y collecter du bois - de chauffe surtout (photo $7 \mathrm{~A}$ ), pour la fabrication de la pâte à papier, du bois d'œuvre aussi - et (ou) des produits forestiers non ligneux - résine (photo 7 B), latex... En revanche, ces plantations forestières ou agricoles (pour les hévéas) ne semblent pas rendre de services écosystémiques culturels, du moins sont-elles considérées comme des «forêts économiques» (rừng kinh tế: nom que leur donnent les villageois) et non comme des forêts sacrées. Les recherches sur les représentations associées aux paysages qu'elles forment et sur les services culturels qu'elles pourraient offrir (en lien avec les valeurs esthétiques, voire les fonctions récréatives...) doivent toutefois se poursuivre. 
Photo 7a. Les plantations, sources de services d'approvisionnement

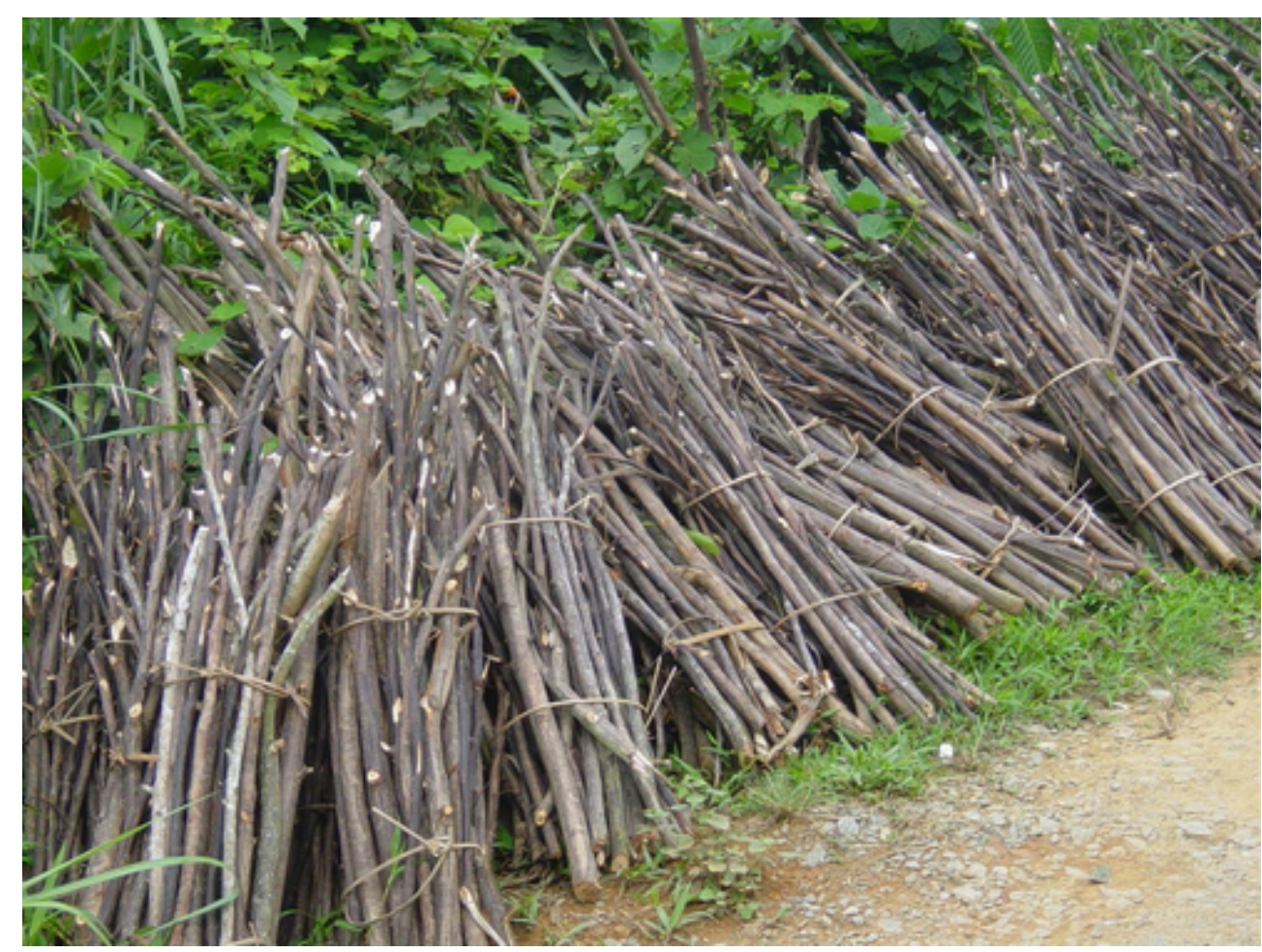

Branches collectées dans les forêts plantées d'acacias pour être utilisées comme bois de chauffe. Source : Amélie Robert, 2007. 


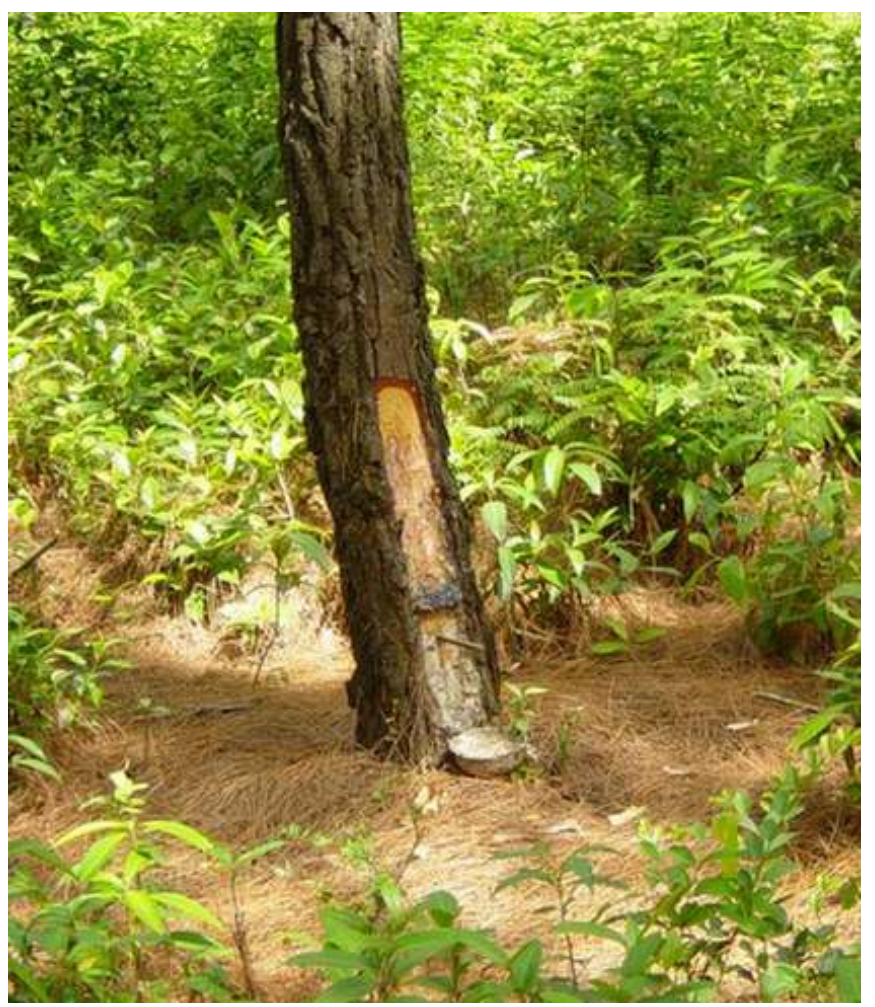

Collecte de la résine des pins (gemmage).

Source : Amélie Robert, 2005.

Au-delà, les plantations présentent des avantages pour l'environnement. Elles permettent le rétablissement rapide d'un couvert forestier (en excluant les plantations d'hévéas qui sont agricoles) sur les versants savanicoles (Amat et al., 2010) et des services écosystémiques divers - y compris de régulation et de support - peuvent dès lors leur être associés (Batra et Pirard, 2015). Les forêts plantées contribuent, par ailleurs, à augmenter la superficie forestière, directement mais aussi indirectement. En effet, elles concourent au recul de l'exploitation des forêts spontanées, dans la mesure où elles fournissent du bois - ou des ressources si ce bois est vendu - aux populations ; elles offrent des services d'approvisionnement qui seraient sinon rendus par les forêts spontanées et conduiraient à la régression de ces dernières (Amat et al., 2010 ; Batra et Pirard, 2015).

Néanmoins, ces plantations offrent de moindres services que les forêts denses spontanées. Plus encore, elles peuvent même présenter des inconvénients pour l'environnement et elles deviennent alors sources de disservices. En effet, équiennes, elles sont souvent exploitées par coupe rase, ce qui facilite l'érosion (Amat et al., 2010). Elles peuvent aussi être un frein à la reconquête spontanée, parce qu'elles remplacent parfois des sylvosystèmes spontanés et ce d'autant plus qu'il existe, pour les acacias, un risque d'invasion (ibid.; Richardson et al., 2015). S'il est admis que les sylvosystèmes spontanés rendent de plus grands services, c'est surtout parce qu'ils se caractérisent par une forte diversité. En effet, selon la thèse de Forest Isbel et al. (2011) : « High plant diversity is needed to maintain ecosystem services. ». Or, les forêts plantées se révèlent « très pauvre[s] au plan de la biodiversité et donc n'ayant rien en commun avec la forêt ombrophile» (Roche et De Koninck, 2001). Il est vrai qu'elles sont monospécifiques 
mais, pour autant, nous l'avons vu, elles rendent des services, au moins d'approvisionnement, aux montagnards et elles leur apportent des ressources. Les services rendus peuvent être moindres que pour les forêts denses spontanées. Mais ils sont supérieurs à ceux des sylvosystèmes spontanés appauvris, en l'occurrence les savanes que ces forêts plantées remplacent bien souvent - elles peuvent aussi se substituer à des terres agricoles -, du moins si on considère les ressources en bois. En effet, pour les propriétaires, les plantations permettent non seulement de satisfaire leurs besoins mais aussi d'en tirer des revenus, par la vente du bois. Pour autant, il est vrai qu'en raison de leur monospécificité, de l'uniformité de leurs arbres, les forêts plantées présentent une vulnérabilité accrue aux fléaux (maladies, incendies... ; Amat et al., 2010). Des tentatives existent toutefois pour diversifier les espèces présentes dans ces forêts, en faveur notamment des espèces locales, introduites en sous-étage des acacias par exemple (photo 8). De telles plantations pourraient offrir davantage de services et concourir plus encore au recul de l'exploitation des forêts spontanées puisqu'elles offriraient du bois utilisable pour la construction - principal facteur de régression des forêts spontanées -, ce qui n'est pas le cas des plantations actuelles, du moins celles d'acacias, qui sont en plein essor.

Les paysages actuels sont bien différents de ceux d'avant-guerre. Les ethnies minoritaires ont dû s'adapter à ce nouveau contexte, modifier leurs pratiques ancestrales pour lesquelles l'arbre spontané était central. La diversité végétale s'est réduite ; les services associés ont évolué.

Photo 8. Tentative de plantation d'une espèce locale (Hopea odorata) en sous-étage des acacias

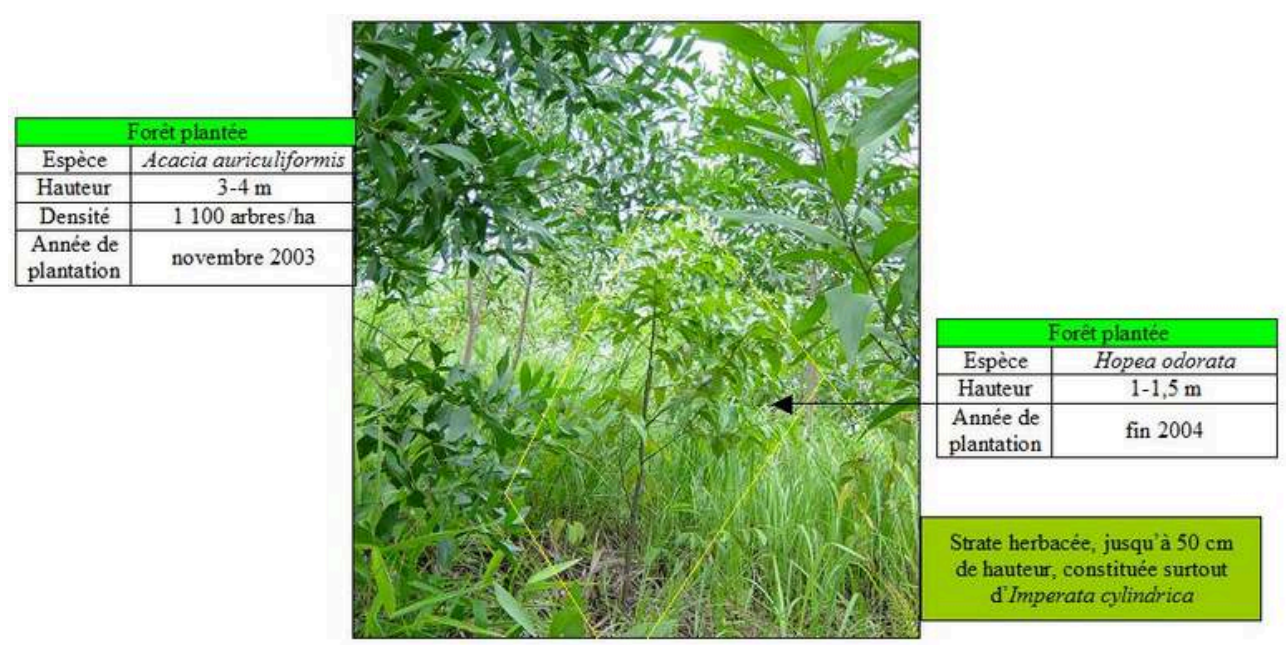

Source : Amélie Robert, 2011.

\section{Conclusion et discussion}

En un peu plus d'un siècle, la place de l'arbre dans les paysages des montagnes de Thừa Thiên Huế a profondément évolué. Sa présence s'est réduite en nombre, en diversité spécifique, sous le poids des pratiques militaires pendant la guerre mais aussi des pratiques civiles avant et surtout après-guerre (Robert, 2011 et 2016), qui ont engendré une accélération du « recul de la forêt » (De Koninck, 1997). La présence de l'arbre s'est 
aussi transformée : avec l'apparition, l'essor même, des plantations depuis les années 1990, il n'est plus seulement spontané, il est aussi planté.

Avant-guerre, le mode de vie, les pratiques, tant forestières qu'agricoles, les croyances des ethnies minoritaires étaient intimement liés aux sylvosystèmes, peuplés alors de ligneux spontanés divers. Les montagnards vivaient ainsi « en osmose » avec les forêts spontanées (Robert, 2011) qui leur offraient «mille ressources» (Gourou, 1940), étant entendu que les savanes ajoutaient à la diversité, y compris arborée, autant qu'aux services rendus. Pendant la guerre et surtout après-guerre, les paysages se sont transformés et les montagnards sont contraints de s'adapter, d'autant plus qu'ils sont sédentarisés pour la plupart dès le lendemain du conflit. Le "recul de la forêt» spontanée, sous le poids des facteurs anthropiques (guerre, exploitation forestière, progression de l'agriculture), a ainsi des incidences sur la biodiversité autant que sur les usages et services rendus aux ethnies minoritaires. Pour enrayer ce recul, la solution adoptée, encouragée par les autorités, est la plantation de forêts. On aboutit ainsi à de nouveaux sylvosystèmes qui offrent eux aussi des services écosystémiques, certes moins importants que les forêts spontanées. Monospécifiques, ils contredisent le lien entre arbre et biodiversité, d'autant plus qu'il existe un risque d'invasion par les acacias. Les paysages arborés ne sont ainsi pas toujours synonymes d'une plus grande richesse spécifique, encore qu'il faudrait aussi prendre en compte la diversité végétale associée aux forêts plantées, présente en sous-étage.

Par cet exemple, on perçoit qu'il existe une diversité de paysages arborés, avec des caractéristiques physiques propres, dont une plus ou moins grande diversité d'espèces végétales. Il en existe aussi une grande variété d'un point de vue plus subjectif, au regard des représentations qui y sont associées: le regard porté par les ethnies minoritaires sur les forêts spontanées diffère par exemple de celui de l'ethnie majoritaire kinh (Robert, 2011). Et il en résulte une variation dans les services écosystémiques associés à ces paysages arborés ; la prise en compte du rôle de l'homme dans l'identification de ces services est contestée par certains (Rives et al., 2016) ${ }^{6}$ mais la notion est éminemment anthropocentrée et il apparaît dès lors difficile de faire abstraction de ce rôle. On perçoit là la complexité des interrelations entre l'homme et la nature, ici entre les sociétés et les paysages arborés. Ces paysages varient dans le temps mais aussi dans l'espace et ce d'autant plus que les représentations et les services qui y sont associés diffèrent aussi. On le remarque pour les paysages arborés issus de plantations, avec en filigrane des différences de représentations de la nature: «Les modèles occidentaux privilégient le concept d'une nature non modifiée par l'homme. De tels modèles ne s'ajustent pas aux représentations paysannes en milieu tropical, qui s'accommodent plus aisément d'une nature domestiquée et susceptible d'assurer les besoins vitaux. En outre, la vitesse de croissance, telle celle des eucalyptus, peut constituer un attrait auprès des populations rurales.» (Tassin, 2011.) Les représentations et services associés aux forêts plantées, plus largement aux paysages arborés, voire à la nature, sont ainsi fonction des cultures. On constate au Viet Nam, où ces forêts plantées sont considérées positivement par les villageois comme des « forêts économiques». Les recherches doivent se poursuivre sur ce lien entre paysages arborés, représentations et services, notamment sur les représentations et services associés à ces forêts, surtout sur leurs services culturels. 


\section{BIBLIOGRAPHIE}

Alexandre, F. et Génin, A., Géographie de la végétation terrestre. Modèles hérités, perspectives, concepts et méthodes, Paris, Armand Colin, 2012, 304 p.

Amat, J.-P., Dorize, L. et Le Chœur, C., Éléments de géographie physique, Paris, Éditions Bréal, 2008, $463 \mathrm{p}$.

Amat, J.-P., Phùng Tửu, B., Robert, A., Trần Hữu, N., « Can fast-growing species form high-quality forests in Vietnam, examples in Thừa Thiên Huế province ", Bois et Forêts des Tropiques, $n^{\circ}$ 305(3), 2010, p. 67-76.

Arnauld de Sartre, X., Castro, M., Dufour, S., Oszwald, J. (dir.), Political ecology des services écosystémiques, Bruxelles, P.I.E. Peter Lang S.A., coll. « EcoPolis », $\mathrm{n}^{\circ} 21,2014,288$ p.

Batra, P., Pirard, R., « Is a typology for planted forests feasible, or even relevant ? », CIFOR infobriefs, $\mathrm{n}^{\circ}$ 121, May , 2015, 8 p.

Bergonzini, J.-C. et Lanly, J.-P., Les Forêts tropicales, Paris, Cirad-Karthala, 2000, 164 p.

Chabert-Ostland, de A. et Gallois, L., Atlas général de l'Indochine française, Hanoi-Haiphong Imprimerie d'Extrême-Orient, 1909, 169 cartes et plans.

Condominas, G., Nous avons mangé la forêt (1957), Paris, Mercure de France, 1974 et 2003, 506 p.

Daily, G. C., Nature's Services : Societal Dependence on Natural Ecosystems, Washington D. C., Island Press, 1997, $392 \mathrm{p}$.

Da Lage, A. et Métailié, G. (dir.), Dictionnaire de biogéographie végétale, Paris, CNRS Éditions, Paris, 2000,579 p.

Đặng Nghiêm Vạn et al., Les Ethnies minoritaires du Vietnam, Hanoi, Éditions Thế Giới, 2000, 308 p.

De Koninck, R., Le Recul de la forêt au Việt Nam, Ottawa, Centre de recherches pour le développement international, 1997, 99 p.

Doray, B et Doray, C, « Le chamanisme en vallée d'A Lưới », dans Maître, J. (dir.), Việt Nam central. Renaissance de la vallée d'A Lưới après les bombes américaines et l'agent orange (1961-2011), Paris, L'Harmattan, 2013, p. 233-256.

Fisher, B., Turner, R. K., « Ecosystem services : classification for valuation », Biological Conservation, $\mathrm{n}^{\circ}$ 141(5), 2008, p. 1167-1169.

Gourou, P., L'Utilisation du sol en Indochine française, Paris, Publications du Centre d'études de politique étrangère, 1940,466 p.

Houzard, G., « Sylvosystème et sylvofaciès : essai d'étude globale du milieu forestier », Phutosociologie et foresterie. Colloques phytosociologiques, t. XIV, 1985, p. 231-236.

Huynh Thu Ba (dir.), People, land and resources in the Central Truong Son landscape, rapport $\mathrm{n}^{\circ}$ $5 \mathrm{du}$ programme Central Truong Son Initiative, Hanoi, WWF Indochina, 2003, 68 p.

Isbel, F. et al., « High plant diversity is needed to maintain ecosystem services ", Nature, vol. 477, 2011, p. 199-202.

Loreau, M., « Par-delà l'éthique et l'économie, l'homme au cœur de la biodiversité », dans Roche, P., Geijzendorffer, I., Levrel, H., Maris, V. (dir.), Valeurs de la biodiversité et services écosystémiques. Perspectives interdisciplinaires, Versailles, éditions Quæ, 2016, p. 79-88. 
Mellac, G. M., « Des forêts sans partage : dynamique de l'espace et utilisation de ressources dans un district de montagnes au Nord Việt Nam ", thèse présentée sous la direction de Rossi, G., académie de Bordeaux, iniversité Michel Montaigne - Bordeaux III, 2000, 579 p.

Millennium Ecosystem Assessment (MEA), Ecosystems and Human Well-being : Synthesis, Washington DC, Island Press, 2005, 155 p.

Nambiar, EK. S., Harwood, C. E., Nguyen, D. C., « Acacia plantations in Vietnam : research and knowledge application to secure a sustainable future », Southern Forests : a Journal of Forest Science. Sustaining the Future of Acacia Plantation Forestry, vol. 77, $\mathrm{n}^{\circ}$ 1, 2015.

Nguyê̂n Nghĩa Thìn et Mai Văn Phô (dir.), Đa dạng sinh học hệ nấm và thực vật - Vườn quốc gia Bạch Mã [Biodiversité des champignons et de la flore - Parc national de Bạch Mã], Bộ Khoa học, Công nghệ và Môi trường [ministère de la Science, de la Technologie et de l'Environnement, Nhà xuất bản nông nghiệp (Maison d'édition de l'agriculture), Hà Nội, 2003, 404 p.

Nguyễn Thu Hương, «Le droit coutumier des minorités ethnolinguistiques », dans Maître, J. (dir.), Việt Nam central. Renaissance de la vallée d'A Lưới après les bombes américaines et l'agent orange (1961-2011), Paris, L'Harmattan, 2003, p. 217-232.

Richardson, D. M., Le Roux, J. J., Wilson, J. RU., « Australian acacias as invasive species : lessons to be learnt from regions with long planting histories ", Southern Forests : a Journal of Forest Science. Sustaining the Future of Acacia Plantation Forestry, vol. 77, n 1, 2015.

Rives, F., Pesche, D., Méral, P. et Carrière, S. M., « Les services écosystémiques : une notion discutée en écologie ", dans Méral, P. et Pesche, D. (dir.), Les Services écosystémiques. Repenser les relations nature et société, Versailles, éditions Quæ, 2016, p. 53-74.

Robert, A., « At the Heart of the Vietnam War : Herbicides, Napalm and Bulldozers Against the A Lưới Mountains »/ «Au cœur de la guerre du Việt Nam : herbicides, napalm et bulldozers contre les montagnes d'A Lưới », Journal of alpine research/ Revue de géographie alpine, nº 104-1, mai 2016, URL : http://rga.revues.org/3266

Robert, A., « Dynamiques paysagères et guerre dans la province de Thừa Thiên Huế (Việt Nam central), 1954-2007 - Entre défoliation, déforestation et reconquêtes végétales ", thèse de doctorat soutenue le 3 décembre sous la direction de J.-P. Amat, université Paris-Sorbonne, 2011, 1172 p. + atlas (159 p.).

Roche, Y. et De Koninck, R., «Les enjeux de la déforestation au Vietnam », Vertigo, vol. 3, $n^{\circ} 1$, octobre 2001, URL : https://vertigo.revues.org/4113

Simon, L., « De la biodiversité à la diversité : les biodiversités au regard des territoires ", Annales de géographie, $\mathrm{n}^{\circ} 651,2006 / 5$, p. 451-467, URL : http://www.cairn.info/revue-annales-degeographie-2006-5-page-451.htm

Servain-Courant, S. et Yengué, J. L., « Les forêts du Val de Loire, maillons dans un paysage emblématique : Bréhémont en Indre-et-Loire », dans Corvol, A. (dir.), Forêt et Paysage. Xe-XxI siècle, Paris, L'Harmattan, 2011, p. 131-140.

SNNVPTNT (Sớ Nông Ngiệp và Phát Triển Nông Thôn [service de l'Agriculture et du Développement rural]), Báo cáo lâm nghiệp. Báo cáo thực trang và các vân đề lâm nghiệp tỉnh Thừa Thiên Huế [Rapport forestier. Situation et problèmes forestiers de la province de Thừa Thiên Huê], juin, Huế, non publié, 2002, 55 p.

Tallis, H. et al., “A global system for monitoring ecosystem service change”, Bioscience, $\mathrm{n}^{\circ}$ 62(11), 2012, p. 977-986 
Tassin, J., «Les plantations forestières industrielles à l'épreuve des représentations de la nature ", Bois et Forêts des Tropiques, n 309 (3), 2011, p. 9-16

\section{NOTES}

1. Le Việt Nam compte 54 ethnies, dont une majoritaire, l'ethnie kinh, peuple de plaine.

2. Ceci est également souligné par «Wallace [qui] considère que les services supports et les services de régulation ne sont pas directement attendus par les humains " (Rives et al., 2016). Wallace, K. J., "Classification of ecosystem services: Problems and solutions", Biological Conservation, vol. 139, $\mathrm{n}^{\circ} 3-4$, October 2007, p. 235-246.

3. Lors des entretiens, les montagnards ont pu mentionner des temps de jachère plus courts mais probablement en raison de confusions avec la situation actuelle.

4. On considère que, $\mathrm{du}$ fait de leur mise en culture, ces sylvosystèmes deviennent des agrosystèmes : c'est alors l'activité agricole qui guide le fonctionnement de ces écosystèmes. Mais on peut aussi considérer que cet état n'est que provisoire et que l'écosystème conserve son statut de sylvosystème, qu'il retrouvera quoi qu'il en soit, d'autant plus que des arbres demeurent souvent présents. Pour autant, on ne parlera pas d'agroforêt car ces arbres ne subsistent que parce qu'ils sont trop gros pour être abattus. Par ailleurs, le terme d'agroforêt s'applique plutôt à des cultures sédentaires, incluant des cultures pérennes, selon la définition donnée par Antoine Da Lage et Georges Métailié (2000).

5. Le Việt Nam n'est pas le seul pays concerné par l'extension des surfaces plantées d'acacias puisque le phénomène se généralise en Asie du Sud-Est. David M. Richardson et al. (2015) évoque ainsi "The massive extent of recent plantings of Australian Acacia species in South-east Asia".

6. Dans la définition des services écosystémiques, plusieurs courants existent et certains considèrent en effet que ne doivent être pris en compte que les services produits par les écosystèmes eux-mêmes, sans intervention de l'homme: "Certains auteurs estiment que les services écosystémiques sont de nature écologique (Fisher et Turner, 2008). Ainsi, dans la mise en œuvre de l'approche par les services, les études qui proposent une évaluation des services se limitent le plus souvent à évaluer un potentiel biophysique, sans tenir compte de l'existence d'un bénéficiaire ou d'une demande (Tallis et al., 2012). » (Rives et al., 2016). Des auteurs vont même jusqu'à considérer "uniquement les services issus des écosystèmes naturels " (ibid.), comme Daily (1997). À l'inverse, "d'autres auteurs estiment que les activités humaines peuvent contribuer à la production de services écosystémiques » (Rives et al., 2016).

\section{RÉSUMÉS}

L'article interroge la place de l'arbre dans les paysages des montagnes du centre du Việt Nam, son évolution depuis la fin du XIX ${ }^{\mathrm{e}}$ siècle, en considérant la diversité des espèces végétales et les services associés - d'approvisionnement et culturels selon la terminologie du Millenium Ecosystem Assessment (MEA, 2005). La méthodologie mêle connaissances endogène et exogène des paysages, en se fondant sur l'analyse de cartes d'occupation des sols (réalisées à partir de sources primaires), de relevés de terrain et d'entretiens avec les populations autochtones. L'objectif est d'identifier les rapports entre l'arbre et les sociétés montagnardes, en interrogeant 
les liens entre paysages arborés, diversité et services, de manière dynamique. Le travail met en avant une évolution majeure, entre l'avant et l'après-guerre - sans que le conflit en soit la seule cause. Jusqu'à la guerre (1945-1975), l'arbre spontané occupe une place centrale dans les paysages. Peuplant des formations végétales surtout denses, il se caractérise par une forte diversité et de nombreux services sont associés aux écosystèmes qu'il forme. Mais les bouleversements de la guerre puis l'accélération du recul de la forêt transforment les paysages. L'arbre spontané se raréfie et cède la place à l'arbre planté. Les pratiques évoluent, les services écosystémiques rendus aussi : les plantations offrent des ressources et sont alors considérées comme des «forêts économiques » mais non comme des forêts sacrées, un statut réservé aux sylvosystèmes spontanés.

The article investigates the role of the tree in mountain landscapes in the central region of Viet Nam and its evolution since the end of the 19th century, by considering the diversity of plant species and the associated services - "supporting and cultural services", according to the terminology of the Millenium Ecosystem Assessment (MEA, 2005). The methodology associates endogenous and exogenous knowledge of the landscapes, based on an analysis of land-use maps (produced from primary sources), field surveys and interviews of indigenous populations. The objective is to identify the relations between trees and mountain communities by conducting a dynamic investigation of the links between forest landscapes and the various services. The research reveals a major change which occurred between the pre and post-war periods - without the conflict being the sole cause. Until the war (1945-1975), spontaneous tree growth occupied a central role in the various landscapes. Populating essentially dense plant formations, it presents a high degree of diversity and many services associated with the ecosystems it forms. But the turmoil caused by the war and the accelerating loss of forest resources has transformed landscapes. Fewer trees grow spontaneously and these are being replaced by planted trees. Practices are changing, the ecosystemic services rendered are also evolving; plantations provide resources and are therefore considered as economic assets and woodlands are no longer as sacred forests, a status reserved to spontaneous sylvosystems.

\section{INDEX}

Mots-clés : paysages, biodiversité, services écosystémiques, Việt Nam, ethnies minoritaires

Keywords : landscapes, biodiversity, ecosystemic services, Viet Nam, ethnic minorities

\section{AUTEUR}

\section{AMÉLIE ROBERT}

Amélie Robert est géographe et enseignante contractuelle à l'université de Tours - UMR Citeres. Ses recherches portent sur les paysages et leurs dynamiques, les services écosystémiques, les interrelations homme-nature, les représentations de la nature, dans différents milieux (urbains et surtout ruraux : sylvosystèmes et agrosystèmes), sur des terrains variés (Việt Nam, France, Burkina Faso).

amelie.robert[at]univ-tours[dot]fr 